\title{
The influence of head contour and nose angle on the perception of eye-gaze direction
}

\author{
STEPHEN R. H. LANGTON, HELEN HONEYMAN, and EMMA TESSLER \\ University of Stirling, Stirling, Scotland
}

\begin{abstract}
We report seven experiments that investigate the influence that head orientation exerts on the perception of eye-gaze direction. In each of these experiments, participants were asked to decide whether the eyes in a brief and masked presentation were looking directly at them or were averted. In each case, the eyes could be presented alone, or in the context of congruent or incongruent stimuli. In Experiment 1A, the congruent and incongruent stimuli were provided by the orientation of face features and head outline. Discrimination of gaze direction was found to be better when face and gaze were congruent than in both of the other conditions, an effect that was not eliminated by inversion of the stimuli (Experiment 1B). In Experiment 2A, the internal face features were removed, but the outline of the head profile was found to produce an identical pattern of effects on gaze discrimination, effects that were again insensitive to inversion (Experiment 2B) and which persisted when lateral displacement of the eyes was controlled (Experiment 2C). Finally, in Experiment 3A, nose angle was also found to influence participants' ability to discriminate direct gaze from averted gaze, but here the effect was eliminated by inversion of the stimuli (Experiment 3B). We concluded that an image-based mechanism is responsible for the influence of head profile on gaze perception, whereas the analysis of nose angle involves the configural processing of face features.
\end{abstract}

Gaze direction represents a biologically significant stimulus that demands rapid and precise discrimination. Indeed, researchers have long been interested in our particular sensitivity to eye direction and the social significance of gaze behaviors. However, there has been rather less interest in the perception of head orientation, despite evidence suggesting that head angle can influence the perception of gaze (Anstis, Mayhew, \& Morley, 1969; Cline, 1967; Gibson \& Pick, 1963; Maruyama \& Endo, 1983, 1984; Wollaston, 1824, as cited in Bruce \& Young, 1998). One exception to this is the work of Wilson, Wilkinson, Lin, and Castillo (2000), who have suggested that humans make use of two cues to determine head orientation: deviation of head profile from bilateral symmetry and the angle of deviation of the nose from vertical. The goal of the present article was to combine the research on head perception with that of gaze perception to determine whether either or both of these cues to head orientation influence the perception of eye-gaze direction.

This research was supported, in part, by Nuffield Foundation Research Bursary NUF-URB/00166/G awarded to S.R.H.L. and H.H. We thank Caroline Carson for her help in running Experiment 2C and both Hugh Wilson and an anonymous reviewer for their helpful comments on an earlier version of this manuscript. Correspondence should be addressed to S.R.H. Langton, Department of Psychology, University of Stirling, Stirling FK9 4LA, UK (e-mail: srhl1@stirling.ac.uk).

Note-This article was accepted by the previous editorial team, headed by Neil Macmillan.

\section{Gaze Perception}

Another's eyes provide a rich source of social information concerning, for example, their owner's disposition toward you, their current emotional state, or whether it's your turn to speak in a conversation (for reviews, see BaronCohen, 1995; Kleinke, 1986). However, the eyes also signal another biologically significant piece of information: the direction in which another's attention is directed. Humans and most other species tend to look at things in their environment which are of immediate importance to them; so you might be rewarded with another's gaze because of a lover's affection or perhaps because you look like a hearty meal. On the other hand, a shift in another's gaze away from you may signal the approach of a predator, prey, or an attractive conspecific (see Byrne \& Whiten, 1991). Therefore, an efficient ability to detect a mutual gaze and to compute precisely where another's eyes are directed offers significant adaptive advantages. Indeed, research has shown that we are very efficient at searching for a direct gaze among averted gaze distractors - the "stare-in-the-crowd" effect (von Grünau \& Anston, 1995) - while our particular sensitivity to gaze direction has been well established (Anstis et al., 1969; Cline, 1967; Gibson and Pick, 1963). Cline (1967), for example, found that humans could detect gaze deviations of just $1.4^{\circ}$ at a distance of just over $1 \mathrm{~m}$. Similarly, Anstis et al's research indicated that humans can detect a displacement of the iris by as little as $1.8 \mathrm{~mm}$ from the same viewing distance. Moreover, there is some suggestion that this peculiar sensitivity may arise - at least in part-from the operation of functionally specific neural mechanisms (e.g., Campbell, Heywood, Cowey, Regard, 
\& Landis, 1990; Heywood \& Cowey, 1992; Hoffman \& Haxby, 2000; Perrett et al., 1985).

In terms of the cues we use to determine another's gaze direction, researchers have traditionally emphasized the spatial or geometric information present within the eye region (e.g., Anstis et al., 1969). So, for example, the high contrast of the limbus (the junction between the sclera and the iris) could be easily located and compared with a fixed feature such as the corner of the eye (the canthus) or the nose. This would give a measure that is proportional to the angle of rotation of the eyeball in the head. However, there are other plausible nonspatial accounts of gaze perception. Watt (1999; see Langton, Watt, \& Bruce, 2000), for example, has argued that the cue to gaze direction might be the contrast in luminance between the two parts of the sclera on either side of the iris, making eye direction a simple measurement to perform on the image of the eye. In support of this account, Watt found that sensitivity to gaze direction did not vary with viewing distance up to a cutoff point beyond which, presumably, the relevant luminance cues could not be resolved (see also Lord \& Haith, 1974). An account based on the geometry of the eye, on the other hand, would predict a deterioration in performance with increased viewing distance.

The results of a recent study by Ricciardelli, Baylis, and Driver (2000) could also be interpreted as offering support for an image-based account. They showed that judgments of gaze direction were highly impaired when the normal contrast polarity of the eyes was reversed so that the sclera appeared to be much darker than the iris. In a similar way, Sinah (2000) contrived the "Bogart illusion" in which contrast negation of a photograph of the eponymous actor's face caused an apparent reversal of his gaze direction. Finally, in Ando's "bloodshot illusion," a bias in participants' gaze judgments was induced by darkening one side of the sclera without shifting the actual location of the iris (e.g., Ando, 2002). Of course, neither contrast negation nor darkening of the sclera affect the spatial relationships between the features of the eye, suggesting that a geometrical mechanism cannot be entirely responsible for normal judgments of gaze direction.

\section{Perception of Head Orientation}

Logically, determination of another's direction of gaze must be based not only on the angle of rotation of the eyeball-however it is computed - but also on the direction in which the head is oriented (Wilson et al., 2000; but see Langton et al., 2000). For example, if the iris is located close to the left corner of a gazer's eye, this might mean that the gazer is looking to your (the viewer's) right, but if - in addition - his or her head is rotated to your left, his or her gaze might then be oriented directly into your eyes.

The importance of head orientation as a cue to attention direction is evident in research in developmental psychology, comparative studies with nonhuman primates, and recent experimental work with human participants. Infants are able to follow a change in their mothers' head and eye orientation from 3 to 6 months of age (Butterworth \& Jarrett, 1991; Scaife \& Bruner, 1975), but it is not until 14 to 18 months that they show any indication of following the eyes alone (Moore \& Corkum, 1998). Prior to this, it seems that children actually ignore the orientation of the eyes and simply use the position of the head as an attention-following cue (Corkum \& Moore, 1995). By and large, nonhuman primates - the nonape species in particular - also use head orientation as the primary cue to another individual's direction of attention (e.g., Emery, Lorincz, Perrett, Oram, \& Baker, 1997; Itakura \& Anderson, 1996). Experimental studies with human participants have indicated that head cues are able to trigger rapid and reflexive shifts of a viewer's spatial attention (Langton \& Bruce, 1999) and are very difficult to ignore, even when the viewer attempts to respond to directional information presented auditorily (Langton, 2000; Langton \& Bruce, 2000). Finally, single-cell recordings of activity in the STS region of the macaque brain have revealed cells that are responsive to certain head orientations and body postures as well as to directions of eye gaze (e.g., Perrett et al., 1985).

Despite the importance of the head as a cue to the direction of social attention, the perception of its orientation has been the subject of relatively little research. Recently, however, Wilson et al. (2000) investigated humans' thresholds for discriminating head orientation and examined the cues with which we might make this discrimination. Their participants were able to perceive a change in head rotation from a base angle of $0^{\circ}$ or $15^{\circ}$ of as little as $1.9^{\circ}$ and $2.1^{\circ}$, respectively, with mean threshold falling off to $4.9^{\circ}$ for a base head angle of $30^{\circ}$. Furthermore, they showed that these thresholds were not significantly affected by removal of either the internal features or the outline head contour, suggesting that head orientation can be discriminated with either of these two equal-strength cues. Finally, by using surrogate nose and head shapes, Wilson et al. established that, for the internal features, the deviation of nose angle from vertical is the likely source of head orientation information, and that the "external" cue is the deviation of the head contour from bilateral symmetry. To elaborate, when the head is oriented directly at you, its outline contour projects an approximately symmetrical shape about the vertical midline, and a line drawn from the bridge to the tip of the nose will be roughly vertical. As the head rotates, its shape becomes increasingly asymmetrical and the nose angle shifts away from vertical. Wilson et al.'s evidence suggests that the visual system is able to compute these deviations from bilateral symmetry and vertical angle and use them as cues to the orientation of the head.

\section{Influence of Head Angle on Gaze Perception}

Since the pioneering work on gaze perception was carried out in the 1960s, it has been known that the perceived direction of eye gaze can be influenced by the angle of rotation of the head, further attesting to the importance of the head as a cue to attention direction. In general, there 
A

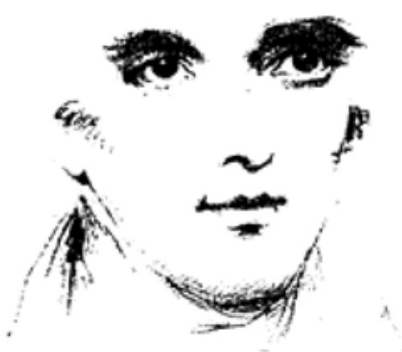

C

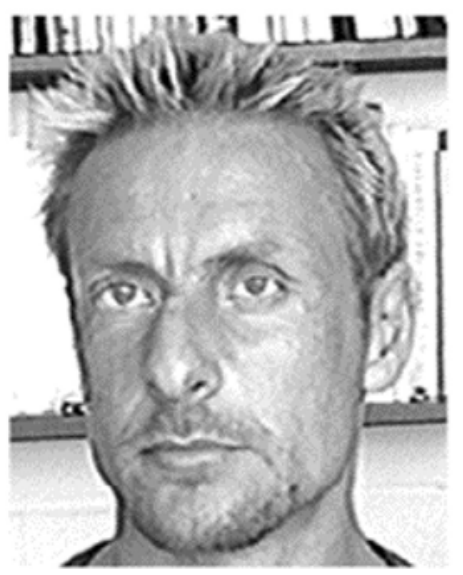

B

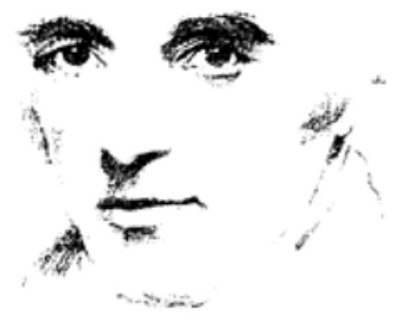

Figure 1. Head orientation influences the perceived direction of gaze. The top two pictures are taken from Wollaston's original paper. Face B seems to be gazing directly at the viewer, whereas face $A$ appears to be looking slightly to the viewer's right. By covering the lower and upper parts of each face, you can see that the eye regions of both are, in fact, identical. The lower two faces illustrate a similar effect with grayscale images. The eye region from face $D$ has been pasted onto face $C$, where the head is rotated slightly to the viewer's left.

seem to be two kinds of perceptual effects. First, under certain circumstances, the perceived direction of gaze can be "towed" toward the orientation of the head. In this case, the direction of gaze is perceived to be somewhere between the angle of the head and the true line of regard of the eyes (Cline, 1967; Maruyama \& Endo, 1983, 1984). This kind of effect was first recorded by William Wollaston as long ago as 1824 and is illustrated in his original drawings reproduced here, along with photographic versions, in Figure 1. The second kind of influence of head angle on the perception of gaze is a kind of "overshoot" or "repulsion" effect in which an error in gaze perception is introduced in the opposite direction to the angle of rotation of the head. For example, imagine someone standing in front of you with his or her head $30^{\circ}$ or so to your right and with his or her eyes either staring straight back at you, or back toward your left shoulder. Apparently, under these conditions, you might perceive his or her eyes to be gazing a little further to the left than they actually are (Anstis et al., 1969; Gibson \& Pick, 1963).
As described in the preceding section, Wilson et al.'s (2000) work suggests that humans are able to use head contour and nose angle to judge head orientation. However, it is not clear whether these are the cues that are actually used in practice and that will interact with information extracted from the eye region to yield the direction of gaze. Thus, the question that concerns us here is whether the cues used to judge head orientation are the same as those that influence the perception of gaze direction. In order to study this, we made use of the Wollaston illusion (see Figure 1). In Experiment 1, we first establish an experimental method for quantifying the illusion. Then in Experiments 2 and 3, we investigate whether head contour and nose angle, respectively, can produce a perceived shift of gaze. The basic design of all experiments was the same. Participants viewed brief masked presentations of eyes which were either directed toward them or were angled slightly to their left or to their right, and their task was simply to decide whether the gaze was direct or averted. These eyes could be placed in one of several contexts: the head 
angle - as signaled by either the head and nose (Experiments $1 \mathrm{~A}$ and $1 \mathrm{~B}$ ), the head outline alone (Experiments $2 \mathrm{~A}, 2 \mathrm{~B}$, and $2 \mathrm{C}$ ), or the nose angle (Experiments $3 \mathrm{~A}$ and $3 \mathrm{~B}$ ) - could be oriented in the same (congruent) or in a different (incongruent) direction to that of the eyes, or the head context could be absent altogether. We then measured how well participants were able to discriminate direct from averted gaze under congruent, incongruent, and absent conditions. With this technique, we were also able to examine whether a direct gaze could be "pulled" to one side, by comparing hit rates (proportion of trials in which participants correctly judged that a direct gaze was indeed oriented at them) in congruent and incongruent conditions. By making this same comparison using false alarm rates (proportion of trials in which an averted gaze was incorrectly judged as being direct) as the dependent measure, we were able to determine whether an averted gaze could be made to appear more direct by an incongruently angled head. Finally, we examined whether each cue could influence the perception of gaze direction when the stimuli were rotated $180^{\circ}$, a manipulation intended to disrupt the configural or spatial/relational processing of faces.

\section{EXPERIMENT 1A}

Experiment 1 was conducted to establish an experimental paradigm for demonstrating that head angle, as signaled by both head contour and nose angle, can influence the perceived direction of gaze. Participants made gaze judgments in the context of grayscale images of heads oriented in congruent or incongruent directions to the eyes. In addition, we examined participants' ability to distinguish direct from averted gaze in the absence of any face context. If head orientation produces a towing effect as in the Wollaston illusion (Figure 1), we would expect performance to be poorer in incongruent than in congruent conditions. Moreover, this reduction in overall discriminability should be caused by both a reduction in hit rates and an increase in false alarm rates in incongruent as opposed to congruent conditions. We predicted that hit rates would decrease because incongruent heads should produce an illusory shifting of a direct gaze, and false alarm rates would increase because averted gazes would tend to be misjudged as being direct when accompanied by an incongruent, as opposed to a congruent, head.

\section{Method}

Participants. Seventeen Open University students attending summer school at the University of Stirling participated in the experiment. All had normal or corrected-to-normal vision.

Materials and Apparatus. Digitized images of eyes gazing straight ahead, approximately $16^{\circ}$ to the left and $16^{\circ}$ to the right, were obtained from grayscale photographs of the face of a male individual with his head oriented forward. These images all had the same shape (see Figure 2 ) and measured $3.8^{\circ}$ wide $\times 1.3^{\circ}$ of visual
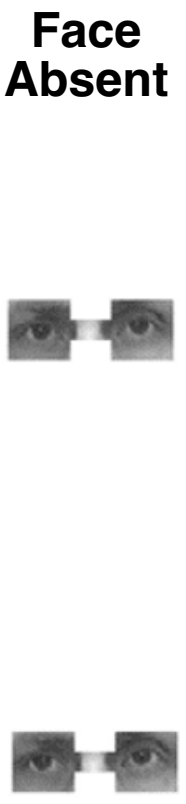

Face Congruent
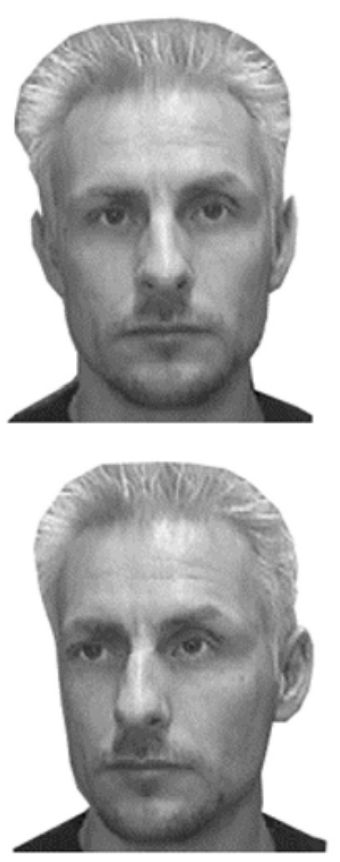

Face Incongruent
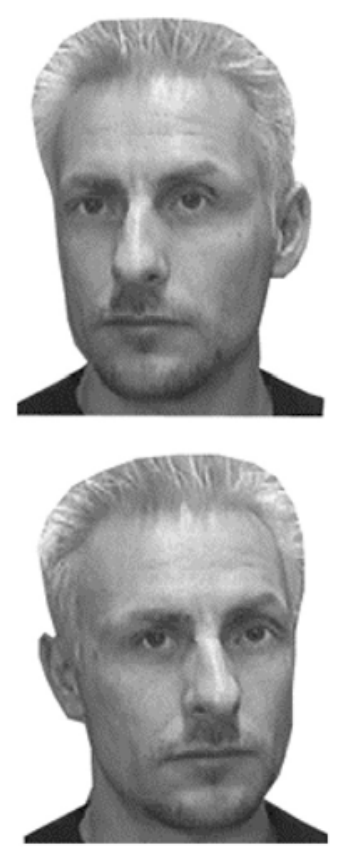

Figure 2. Reproductions of some of the stimuli used in Experiments 1A and 1B. The left column contains stimuli in the face-absent condition; the middle column, stimuli in the facecongruent condition; and the right column, stimuli in the face-incongruent condition. The upper row of stimuli have direct gazes, and those in the lower row, gazes averted to the left. 
angle in height. In addition, full-face images of the same individual were obtained with his head oriented straight ahead, $16^{\circ}$ to the left, and $16^{\circ}$ to the right. These images subtended $7.1^{\circ}$ of horizontal and $9.5^{\circ}$ of vertical visual angle. The materials used in the congruent conditions of the experiment were obtained by pasting the three gaze stimuli onto the appropriately oriented head stimuli with Adobe Photoshop software. Thus, the leftward gaze from the full-face image was pasted onto the image of the head oriented to the left, and so forth. A blending tool was then used to eliminate sharp lines so that the resulting face appeared smooth. Incongruent images were obtained by pasting the straight-ahead gaze stimuli onto the left and right head images, and by pasting the left and right gaze stimuli onto right and left head images, respectively. In this way, the same direct and averted-gaze stimuli could be presented alone, in the context of a congruent head orientation, or an incongruent head orientation. Examples of the experimental stimuli are shown in Figure 2.

The experimental stimuli were presented at fixation on a white background. Each was preceded by a black fixation cross comprising vertical and horizontal lines measuring $0.6^{\circ}$, and followed by the presentation of a pattern mask. This measured $7.6^{\circ} \times 9.5^{\circ}$ and was created by pixelating the full-face image, using Photoshop's pointillize tool with cell size set to 16 . All stimuli in this and subsequent experiments were presented with SuperLab software (Cedrus Corp.) on a Macintosh G3 computer. Participants were seated $0.6 \mathrm{~m}$ from a $15-$ in. color monitor set to grayscale.

Design. The direct and averted-gaze stimuli were presented in a within-subjects design with one factor: head context. The head was absent, congruent, or incongruent with the gaze direction. On each trial, participants were asked to decide whether the eyes were averted or were looking at them, and their proportions of hits and false alarms under each condition were recorded. From these an $A^{\prime}$ scorea measure of participants' ability to discriminate direct from averted gaze - was computed for each of the three conditions and served as the main dependent variable in the experiment.

Procedure. Trials began with the presentation of the fixation cross, which remained on the screen for $1,000 \mathrm{msec}$. This was then replaced by a $140-\mathrm{msec}$ presentation of one of the gaze stimuli, followed by the pattern mask, which remained on the screen for $200 \mathrm{msec}$. The screen then went blank and remained so until the participants responded. Participants were asked to judge whether the eyes were averted or were looking directly at them by pressing, respectively, either the " $m$ " or the " $z$ " key on a standard keyboard. They were asked to respond as accurately as possible and to take as long as they needed to respond, because only their accuracy was being recorded. Following a response, a 1,000-msec delay preceded the beginning of the next trial.

Each participant completed 64 trials in each of the three experimental conditions. These comprised 32 direct-gaze stimuli and 16 stimuli with gaze averted to the left and 16 with gaze averted to the right. These were divided into two identical blocks of 96 trials, in which trial presentations were randomized. Prior to the two experimental blocks, participants completed a sequence of 48 practice trials, 16 in each condition with an equal number of direct and averted stimuli.

Table 1

Means and Standard Deviations of $A^{\prime}$ Values, Hit Rates, False Alarm Rates, and $B^{\prime \prime}$ Values Recorded in Each Condition of Experiment $1 \mathrm{~A}$

\begin{tabular}{|c|c|c|c|c|c|c|}
\hline \multirow[b]{3}{*}{ Measure } & \multicolumn{6}{|c|}{ Face Context } \\
\hline & \multicolumn{2}{|c|}{ Absent } & \multicolumn{2}{|c|}{ Congruent } & \multicolumn{2}{|c|}{ Incongruent } \\
\hline & $M$ & $S D$ & $M$ & $S D$ & $M$ & $S D$ \\
\hline Discriminability $\left(A^{\prime}\right)$ & .68 & .18 & .95 & .06 & .21 & .13 \\
\hline Hit rate & .93 & .06 & .93 & .08 & .22 & .18 \\
\hline False alarm rate & .71 & .25 & .11 & .16 & .65 & .16 \\
\hline Response bias $\left(B^{\prime \prime}\right)$ & .39 & .33 & .07 & .45 & -.18 & .36 \\
\hline
\end{tabular}

\section{Results}

In this and all subsequent experiments, hit rates (proportion of direct-gaze trials in which participants made a correct response) and false alarm rates (proportion of averted-gaze trials in which participants indicated gaze was direct) were first computed for each participant under each of the three experimental conditions. Because some participants recorded no misses or false alarms in some conditions, corrected hit and false alarm rates were computed by first adding 0.5 to the number of hits and false alarms, respectively, in each condition and then incrementing the number of trials in each condition by 1 in order to calculate the probabilities. From each pair of corrected hit and false alarm rates in each condition, $A^{\prime}$ and $B^{\prime \prime}$ scores were then obtained, following the procedure outlined by Snodgrass and Corwin (1988). $A^{\prime}$ is a nonparametric measure of discriminability; in other words, it is a measure of how well participants were able to distinguish direct from averted gaze. $B^{\prime \prime}$ is the equivalent nonparametric measure of response bias, which indexes whether participants tended to prefer one response over the other. A $B$ " score of zero represents a neutral bias, and-in our experiments - a negative value of $B^{\prime \prime}$ represents a conservative bias (i.e., the participant tends to respond "averted") and a positive score, a liberal bias (i.e., a tendency to make more "direct" responses).

Mean values of $A^{\prime}$, hit rates, false alarms, and $B^{\prime \prime}$ in each condition of Experiment 1A appear in Table 1. Examination of the $A^{\prime}$ data indicates that participants were well able to discriminate direct from averted gaze in the congruent condition (mean $A^{\prime}=.95$ ), but their performance deteriorated when the face context was removed (mean $A^{\prime}=.68$ ) and deteriorated still further when head angle and gaze direction were incongruent (mean $A^{\prime}=.21$ ).

An analysis of variance (ANOVA) comparing mean $A^{\prime}$ values in the three conditions yielded a significant effect of head context $[F(2,32)=112.34, p<.001]$. Post hoc Newman-Keuls tests $(\alpha=.05)$ confirmed the observations above; participants' ability to discriminate direct from averted gaze was significantly better in congruent than in both incongruent and absent conditions. Moreover, performance was significantly poorer in the incongruent condition than in the absent condition.

Clearly, head context influenced participants' performance. However, this overall effect on discriminability could have originated from one, or both, of two sources: First, when eyes directed straight ahead were placed in the context of a head that was oriented to either the left or right, participants might have perceived the direction of gaze as being pulled in the direction of the head turn; second, an averted gaze directed to a viewer's left, for example, may have been perceived as directed straight ahead when in the context of a head rotated to the right (see Figure 1). The first type of effect (direct gaze being pulled to the left or right) will cause participants to "hit" a smaller proportion of direct gazes in incongruent than in congruent conditions. The second type of effect (an averted gaze being pulled toward the center by a head rotated in the op- 
posite direction) will produce a higher proportion of false alarms (mistakenly responding "direct" to an averted gaze) in incongruent than in congruent conditions. Either, or both, of these effects could have produced the observed decrease in discriminability when head and gaze were oriented in incongruent directions. In order to examine these two possibilities, separate analyses of hit and false alarm rates were undertaken.

From Table 1, it is clear that mean hit rates were much lower in the incongruent $(M=.22)$ than in the congruent $(M=.93)$ condition, which would suggest that a turn of the head produces an illusory shift of a direct gaze. A repeated measures ANOVA comparing mean hit rates across the three context conditions yielded a significant effect $[F(2,32)=211.93, p<.001]$. Furthermore, a planned comparison revealed that the mean hit rate was significantly lower in the incongruent condition than when head and gaze were congruent $[t(32)=17.84, p<.001]$, confirming the observation above.

False alarm rates also differed across the three context conditions. In particular, participants made a higher proportion of false alarm responses in the incongruent condition $(M=.65)$ than in the congruent condition $(M=$ .11 ), suggesting that a head turn was able to make an averted gaze appear to be directed toward the observer. In support of these observations, a repeated measures ANOVA yielded a significant effect of condition $[F(2,32)=49.48$, $p<.001]$, and a planned comparison confirmed that participants made significantly more false alarms in the incongruent than in the congruent condition $[t(32)=7.83$, $p<.001]$.

In order to determine whether any of the face context conditions produced a systematic response bias, $B^{\prime \prime}$ scores in each condition were compared with a score of zerothe $B^{\prime \prime}$ value corresponding to a neutral bias. The $B^{\prime \prime}$ values presented in Table 1 indicate that participants' responses were only slightly biased in congruent and incongruent conditions but that when the face was absent, they tended to set a rather more liberal criterion, resulting in a bias toward responding that gaze was "direct." A series of onesample $t$ tests comparing the mean $B$ " values with zero confirmed these observations. There were no significant biases in congruent or incongruent conditions $(p s>.05)$ but a significant positive bias when the face was absent $[t(16)=4.88, p<.001]$.

\section{Discussion}

The results of this experiment clearly confirm that head context, and its orientation in particular, has an effect on gaze perception. Participants' ability to discriminate direct gaze from averted gaze was significantly poorer when head and gaze were incongruent than when both were oriented in a congruent direction. Moreover, the results suggest that this effect on discriminability can be attributed to illusory shifts of both direct and averted gazes. When the eyes were paired with an incongruent, as compared with a congruent, head, participants were less likely to respond that a direct gaze was actually looking at them. Similarly, a gaze directed to either the viewer's left or the viewer's right was more likely to be misjudged as a direct gaze when paired with a head oriented in the opposite direction than when paired with a congruent head cue. Thus, as with the Wollaston illusion (see Figure 1) and in line with the findings of Cline (1967) and Maruyama and Endo (1983, 1984), it seems that head orientation produces a towing effect on the perceived direction of gaze so that it falls somewhere between the true line of regard of the eyes and the angle of rotation of the head.

However, before concluding that the effect arises as the result of some kind of perceptual illusion, we should perhaps consider some alternative explanations. First, the influence of head angle on gaze discriminability found in this experiment cannot simply be attributed to participants' adopting a strategy of responding, when uncertain, on the basis of the most visually salient cue: head orientation. Although this strategy would indeed produce a reduced rate of "direct" responses (hits) in the congruent condition and a corresponding reduction in overall discriminability $\left(A^{\prime}\right)$ as found in Experiment 1A, it would not produce the observed increase in false alarms observed in the incongruent condition where neither head nor gaze was actually oriented toward the observer.

It is also difficult to attribute the results of Experiment $1 \mathrm{~A}$ to some kind of response competition effect in which information from head and gaze compete more in incongruent than in congruent conditions. First, such effects are only usually apparent when a speeded response is required. In contrast to this, participants in Experiment 1A were asked to respond as accurately as possible and were explicitly told that their response speed was not being recorded. Second, if some kind of response competition effect were operating here, we might expect that in incongruent conditions, participants would respond on the basis of the actual gaze direction on roughly half of the trials and on the basis of the orientation of the head on the other half of the trials. The data do not, however, support such an interpretation. Under this account, the mean hit rate for direct gazes in the incongruent condition would be expected to be roughly .5 , because participants respond on the basis of gaze (direct) and head orientation (averted) in half of the trials. However, the recorded figure was a significantly lower .22 [one sample $t$ test, $t(16)=6.41, p<$ .001]. Participants in Experiment 1A also made a substantial number of false alarm responses to averted gazes in incongruent trials $(M=.65)$. Under a response competition account, this figure would actually be expected to be closer to zero because both head and gaze direction are averted in opposite directions in the incongruent condition. Participants responding randomly on the basis of either cue would therefore rarely make a "direct" (false alarm) response. Of course, the recorded mean false alarm rate of .65 was found to be significantly higher than zero $[t(16)=16.85, p<.001]$, which again argues against a response competition account.

Thus, it seems unlikely that the findings of this experiment can be attributed to some kind of response bias (re- 
sponding to the most salient cue) or to a response competition effect. Instead, the pattern of results obtained here is consistent with observers' perceived direction of gaze being towed toward the angle of the head, making averted gazes appear to be direct and direct gazes appear to be averted.

As noted in the introduction, other researchers have obtained a rather different effect when head and gaze are placed into conflict in photographic images of faces. Rather than the perceived direction of gaze being towed toward the orientation of the head, both Anstis et al. (1969) and Gibson and Pick (1963) noted that gaze direction is perceived to be shifted in the direction opposite to the orientation of the head. This "repulsion" or "overshoot" effect might occur when, say, leftward-gazing eyes in a rightwardoriented head are perceived as more leftward gazing than they appear to be in a frontward-oriented head. Since this kind of combination of eye and head orientation occurs in certain conditions of Experiment 1A (see, for example, the lower right image in Figure 2), we might ask why a similar repulsion effect was not observed in this study. One possibility is that the repulsion effect occurs, not as a direct result of some interaction between head orientation and gaze direction, but because the effect of a head turn is to expose more visible sclera on one side of the eye or the other. Since the relative proportion of sclera on either side of the iris can be used as a cue to gaze direction (Ando, 2002; Watt, 1999), changing this ratio by exposing more sclera might result in an illusory shift in gaze. For example, imagine someone facing you with his or her eyes gazing directly into yours; roughly the same amount of sclera will be visible on either side of each iris. The contrast in luminance between these parts of the sclera will therefore be roughly zero, yielding the percept of a direct gaze. If that person then turns his or her head to your left while maintaining eye contact, proportionately more of the sclera will now be visible on the left side of his or her eyesfrom your point of view - than on the right. Because this luminance configuration ordinarily signals a rightwarddirected gaze, you will therefore erroneously judge the eyes to be oriented slightly to the right. Indeed, the scleral contrast account of gaze perception predicts just this kind of repulsion effect for certain viewing angles of the face (see Langton et al., 2000).

The absence of a repulsion effect in the present experiment can therefore be explained by the fact that the relative proportion of sclera visible on either side of the iris was held constant across all changes of head orientation. This was achieved by cutting leftward- and rightwardfacing eyes from images of frontward-oriented heads and pasting them onto heads with congruent and incongruent angles of rotation. In view of this, we argue that the Wollaston illusion and the towing effects obtained here and elsewhere index some kind of integration between information coding the orientation of the head and the direction of eye gaze, rather than an error introduced as a consequence of how a turn of the head alters one of the cues used to determine gaze direction.
Another notable finding of this experiment was the significant decrease in $A^{\prime}$ when the congruent-head context was removed so that gazes were presented in isolation from the head. This is in line with the results of a study by Vecera and Johnson (1995), who showed that disruption of the face context by scrambling the features of a schematic face significantly reduced participants' ability to distinguish between direct and averted gazes. In our own work (Jenkins \& Langton, 2003), we have also reported that thresholds for gaze judgments were higher when grayscale images of eyes were presented in isolation than when they were presented in the context of an upright face. We suggest that there are at least two possible reasons for this effect, related to the two components necessary for accurate gaze judgments: locating the position of the eye in relation to the head, and combining this with the angle of orientation of the head. First, removal of the face context also removes a good deal of information that might be used in the spatial computation of the location of the eye in relation to the head. However, it would seem that sufficient information remains for this relational computation to be made even after removal of the face context. The location of the iris need only be computed in relation to some fixed part of the head, and the canthus (the corner of the eye) or bridge of the nose would suffice (see Langton et al., 2000). Inspection of Figure 2 reveals that these features remain intact in the face-absent stimuli. Thus, it is more likely that removal of the face context disrupts the second component necessary for accurate gaze judgments: perception of the angle of rotation of the head. With no information available from the head contour or from the angle of deviation of the nose, perception of head angle might well be impaired.

Removal of the face context also had an effect on participants' response bias. More specifically, in the absence of a face context, participants tended to lower their criterion for making a "direct" response. This seems to be a reasonable strategy; with less information with which to make a decision, defaulting to assuming that gaze is directed at you is, adaptively speaking, a "safe" strategy. In other words, it is better to run the risk of making a few false alarms than to miss one occasion when a predator is eyeing you for its next meal.

To summarize, Experiment 1A was successful in inducing a Wollaston-type illusion in our participants. Moreover, the design is such that it allows the size of the effect to be quantified so that we can go on to manipulate the available cues to head orientation and examine the impact of these manipulations on the magnitude of the effect. Before embarking on this, however, we first assess whether or not the effect of head context on gaze discriminability is sensitive to inversion of the stimuli (i.e., rotation through $\left.180^{\circ}\right)$.

\section{EXPERIMENT 1B}

In this experiment, we asked whether the influence of head rotation on gaze perception noted in Experiment 1A 
might be caused by a low-level image-based mechanism or a higher level process perhaps specific to faces. In order to examine this, the gaze and masking stimuli used in the previous experiment were each rotated about $180^{\circ}$ to produce a set of inverted images.

Numerous studies have demonstrated that inversion severely disrupts various aspects of face processing (e.g., Bruce \& Langton, 1994; Diamond \& Carey, 1986; Valentine \& Bruce, 1986; Yin, 1969). For instance, Yin (1969) showed that recognition memory for upright faces was better than that for pictures of houses, airplanes, or schematic men-in-motion, but when all these materials were inverted, performance on the faces became worse than that on the other pictures. At present, it is unclear exactly what causes the inversion effect, but it is generally agreed that it disrupts a mode of processing variously described as configural (e.g., Sergent, 1984), holistic (e.g., Tanaka \& Farah, 1993), relational (e.g., Goldstone, Medin, \& Gentner, 1991), or noncomponential (e.g., Barton, Keenan, \& Bass, 2001). The basic idea is that the encoding of an upright face involves not only processing of information about individual face features (mouth, nose, eyes, etc.) but also processing of information about the spatial arrangement or configuration of these features (e.g., Leder \& Bruce, 2000; for a recent review of configural processing, see Maurer, Le Grand, \& Mondloch, 2002). It is thought that inversion selectively disrupts - or at least has a greater effect on-the encoding of this configural information. Some direct evidence for this comes from work by Leder and Bruce (1998) and Searcy and Bartlett (1996). In these studies, faces were made to look more grotesque (Searcy \& Bartlett, 1996) or distinctive (Leder \& Bruce, 1998) by either manipulating individual face features (e.g., blurring the pupils or darkening the lips) or distorting the relationships between these features (e.g., narrowing the interocular distance). When inverted, faces made distinctive or grotesque by feature changes still appeared to be distinctive or grotesque, whereas faces changed by manipulating the relationship between features looked more like the original, unaltered versions. In other words, these studies suggest that feature information is still encoded in inverted faces, but the encoding of the relationship between these features is disrupted. Furthermore, the idea that inversion has its effect at the perceptual encoding stage of face perception is consistent with studies

Table 2

Means and Standard Deviations of $A^{\prime}$ Values, Hit Rates, False Alarm Rates, and $B^{\prime \prime}$ Values Recorded in Each Condition of Experiment 1B

\begin{tabular}{|c|c|c|c|c|c|c|}
\hline \multirow[b]{3}{*}{ Measure } & \multicolumn{6}{|c|}{ Face Context } \\
\hline & \multicolumn{2}{|c|}{ Absent } & \multicolumn{2}{|c|}{ Congruent } & \multicolumn{2}{|c|}{ Incongruent } \\
\hline & $M$ & $S D$ & $M$ & $S D$ & $M$ & $S D$ \\
\hline Discriminability $\left(A^{\prime}\right)$ & .74 & .24 & .96 & .02 & .22 & .10 \\
\hline Hit rate & .91 & .06 & .93 & .07 & .24 & .14 \\
\hline False alarm rate & .54 & .29 & .08 & .08 & .62 & .25 \\
\hline Response bias $\left(B^{\prime \prime}\right)$ & .37 & .38 & .03 & .62 & -.05 & .44 \\
\hline
\end{tabular}

using event-related brain potentials which have established that inversion exerts consistent effects as early as $170 \mathrm{msec}$ after stimulus presentation (Bentin, Allison, Puce, Perez, \& McCarthy, 1996; Eimer, 2000; Rossion et al., 1999).

There is also evidence that extensive experience with faces may be required to produce the inversion effect, because face recognition by children below the age of 10 is less affected by inversion (Carey \& Diamond, 1977). Indeed, extensive experience with other categories of objects normally encountered in a particular orientation may also make these objects susceptible to the inversion effect. So, for example, Diamond and Carey (1986) demonstrated that dog-show judges' ability to recognize dogs was also disrupted by inversion. The implication is that we have to learn to encode the relevant configural information in order to make within-category discriminations. Encoding this information becomes difficult with stimuli with which we are not familiar, such as upside-down faces.

Regardless of the precise mechanism behind the inversion effect, this manipulation provides a way of discriminating between a low-level image-based account, and a higher level mechanism based perhaps on face-specific (or expertise-specific) configural processing. If the influence of head orientation on the processing of gaze direction is caused by a higher level mechanism concerned with encoding the configural arrangement of face features, we would expect it to be eliminated by inversion of the stimuli. If, on the other hand, the effect emerges much earlier in processing as the result of an interaction of image-based features, it should persist when the stimuli are inverted.

\section{Method}

Participants. Seventeen volunteers attending an Open University residential summer school at the University of Stirling participated in this experiment. All had normal or corrected-to-normal vision.

Materials, Design, and Procedure. These were identical to those of Experiment 1A; however, the gaze stimuli and pattern mask were rotated through $180^{\circ}$.

\section{Results}

Mean $A^{\prime}$ and $B^{\prime \prime}$ values, along with mean hit and false alarm rates in each condition of Experiment 1B, are presented in Table 2. The pattern of results was very similar to that of Experiment 1A. Participants were less able to discriminate direct gaze from averted gaze in the incongruent condition (mean $A^{\prime}=.22$ ) than in the congruent condition (mean $A^{\prime}=.96$ ). Moreover, incongruently angled heads reduced hit rates and increased false alarm rates, compared with heads oriented in congruent directions to the angle of gaze.

A series of repeated measures ANOVAs and follow-up comparisons conducted on the $A^{\prime}$ scores, hit rates, and false alarm rates confirmed the observations above. Head context exerted a significant effect on discriminability scores $[F(2,32)=103.56, p<.001]$ and post hoc NewmanKeuls tests $(\alpha=.05)$ indicated that the differences between all pairs of means were significant. The effect of 
context was also significant for hit rates $[F(2,32)=346.96$, $p<.001]$ and false alarm rates $[F(2,32)=24.46, p<$ $.001]$. Separate planned comparisons of hit rates and false alarms in congruent and incongruent conditions revealed significant differences in both cases [for hit rates, $t(32)=$ 23.08, $p<.001$; and for false alarms, $t(32)=6.51, p<.01$ ].

As with the upright stimuli, participants operated with a positive bias in judging gaze (i.e., they made more "direct" responses) when the head context was absent, but they showed little bias in the other conditions. One-sample $t$ tests confirmed that the mean bias score in the absent condition was significantly greater than zero $[t(16)=4.03$, $p<.01]$ but that participants displayed no significant bias in congruent or incongruent conditions ( $p \mathrm{~s}>.6)$.

\section{Discussion}

The results of Experiment 1B were almost identical to those of Experiment 1A. Even with inverted stimuli, head context produced an effect on gaze discriminability; participants showed reduced $A^{\prime}$ scores with incongruent as opposed to congruent stimuli. Moreover, as in Experiment $1 \mathrm{~A}$, hit rates were lower and false alarms higher when head and gaze were incongruent than when they were congruent. The influence of head angle on the perception of gaze therefore persisted when the face stimuli were inverted, licensing a conclusion that the root of the effect is a low-level image-based mechanism, not a process that is necessarily specific to faces or based on the relational aspects of the gaze/head stimuli.

The findings of this experiment are, however, at odds with those of Maruyama and Endo (1984), whose Wollastonlike illusion was markedly reduced by the inversion of their face stimuli. They concluded that inversion disrupted the configural integration of face features that they thought underpinned the effect. However, their studies differed from ours in at least two important respects, both of which might explain the discrepant findings.

First, Maruyama and Endo (1984) used a finer grained measure of perceived gaze direction: Participants were asked to indicate where they perceived the gaze to be directed by marking a point on a Perspex arc positioned in front of the schematic face. Thus, it is possible that their measure of the Wollaston-like illusion was more sensitive to any effects of inversion than the measure used in our experiments. However, we believe that, given the strength of the illusion found with our stimuli, a finer grained measure would - at best - simply reveal a slightly weaker effect in inverted faces than in upright faces (readers might like to satisfy themselves of the robustness of the illusion by viewing Figures 1 and 2 with the pages turned upside down). Even if the illusion is actually slightly weakened in inverted faces, it still begs the question of why it persists at all under conditions in which the encoding of relations between face features is known to be severely disrupted, and probably particularly so in the brief, masked displays that we have used. The likely explanation is that the effect emerges as the result of an interaction between imagebased features, rather than face-specific representations.
The discrepancy between our findings and those of Maruyama and Endo (1984) is perhaps more likely to rest on a second major difference between the two studies: their use of schematic, as opposed to grayscale, images of faces. Maruyama and Endo (1984) used a circle to represent the outline contour of their schematic faces, even in conditions in which the head was rotated. Their participants were therefore unable to use the overall shape of the head as a cue to head orientation. Instead, they had to rely on two other potential cues: the shape of a line denoting the profile of a nose, mouth, and chin drawn within the circular face frame; and the horizontal displacement of the eyes and the profile shape, again within the circular face outline. These cues were evidently successful in producing the illusion of a rotated head and, in turn, an illusory shift of eye gaze in upright faces. Although these cues were potentially present in the grayscale stimuli used in Experiments $1 \mathrm{~A}$ and $1 \mathrm{~B}$, these images also include what Wilson et al. (2000) regard as one of the strongest cues to head orientation: the shape of the head profile or, more specifically, its degree of deviation from bilateral symmetry. The discrepant findings between our experiments and those of Maruyama and Endo (1984) might therefore have been due to the fact that different cues to head orientation were available in these studies, and that these cues might well have influenced the perception of gaze in rather different ways. The occluding contour formed by the shape of the head, for example, might have been sufficient on its own to exert an effect on the processing of eye-gaze direction, but it might have done so at an early stage in processing that was insensitive to inversion. Cues such as nose angle and eye displacement, on the other hand, might also be capable of influencing the extraction of gaze, but they would do so later in processing as the result of some kind of configural mechanism that $i$ disrupted by inversion. In the remainder of the experiments reported here, we explored some of these issues. In Experiments $3 \mathrm{~A}$ and $3 \mathrm{~B}$, we examined whether a Wollaston-type illusion could be induced by deviations in the angle of the nose. Meanwhile, in Experiments $2 \mathrm{~A}-\mathrm{C}$, we examined whether the outline contour of the head would be sufficient to influence the perception of gaze direction.

\section{EXPERIMENT 2A}

In order to test whether head shape alone is able to influence gaze perception, the face images used in Experiments $1 \mathrm{~A}$ and $1 \mathrm{~B}$ were first subjected to a high-pass filter and then the internal features, apart from the eyes, were removed from the resulting images, leaving only the outline contour of the head. As before, we then examined how well participants were able to discriminate direct gaze from averted gaze under conditions in which the head outline alone was congruent, incongruent, or absent. If head outline is indeed used to perceive head orientation, and this information is then used to influence gaze perception, we would expect the context provided by the head contour to exert an effect on gaze discrimination. 

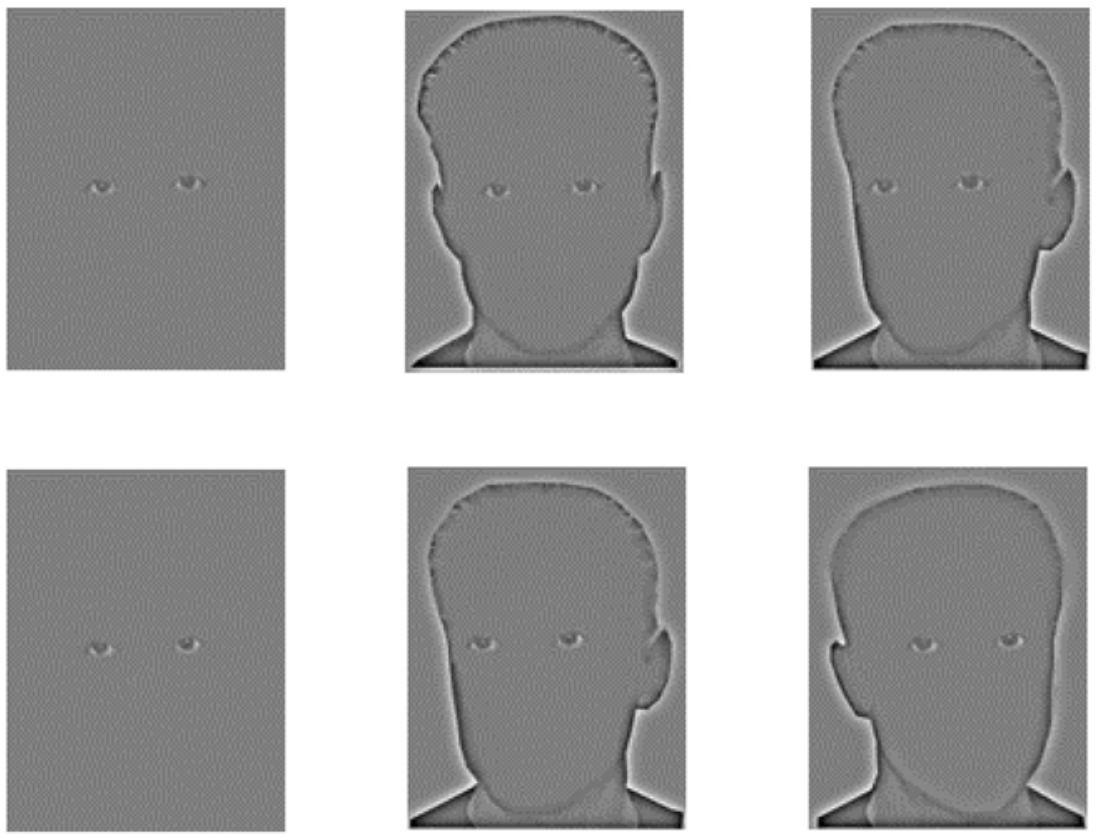

Figure 3. Reproductions of some of the stimuli used in Experiments $2 \mathrm{~A}$ and 2B. The left column contains stimuli in the head-absent condition; the middle column, stimuli in the headcongruent condition; and the right column, stimuli in the head-incongruent condition. The upper row of stimuli have direct gazes, and those in the lower row, gazes averted to the left.

\section{Method}

Participants. Seventeen Open University students drawn from the same population as in Experiment 1 participated in this experiment. Again, all had normal or corrected-to-normal vision.

Materials, Design, and Procedure. In order to create stimuli where only the outline contour of the head could provide information about head angle, the internal features were removed from the original grayscale images of the head directed straight ahead, angled to the left and to the right. This was accomplished in the following way. First, Adobe Photoshop was used to subject each of these three images to a high-pass filter. After filtering, a paintbrush tool was used to replace the internal region of each face with the same gray level as that of the background, leaving only the outline contour of the head visible. This resulted in three separate images of head outline shapes: one angled to the left, one to the right, and a third straight ahead. Next, the stimuli used in the head-absent context condition in Experiment 1A were also subjected to the same high-pass filter, and the paintbrush tool was used to remove any information from the areas surrounding the eyes. The resulting eyes-only images served as stimuli in the head-absent condition of Experiment 2A. Copies of these stimuli were then pasted onto the appropriate head outline im- ages to create the congruent and incongruent stimuli analogous to those used in Experiment 1. Care was taken to ensure that the eyes were pasted onto the identical position, relative to the head outline, as in the original digitized grayscale images. Examples of the stimuli used in each condition of Experiment $2 \mathrm{~A}$ are shown in Figure 3.

The pattern mask used in Experiment 1A was also high-pass filtered and used as the mask in this experiment. Stimuli were presented on a background with the same gray level as the median level of that of the experimental stimuli. All other aspects of the design and procedure remained the same as in Experiment 1A.

\section{Results}

Measures of discriminability $\left(A^{\prime}\right)$ and bias $\left(B^{\prime \prime}\right)$ were calculated as in Experiments $1 \mathrm{~A}$ and $1 \mathrm{~B}$, and the means of these values in each experimental condition are reported in Table 3, along with mean hit and false alarm rates. This table makes clear that the effect of head-outline context on gaze perception was strikingly similar to that of the full face in Experiment 1A. Participants were well able to dis-

Table 3

Means and Standard Deviations of $\boldsymbol{A}^{\prime}$ Values, Hit Rates, False Alarm Rates, and $B^{\prime \prime}$ Values Recorded in Each Condition of Experiment 2A

\begin{tabular}{|c|c|c|c|c|c|c|}
\hline \multirow[b]{3}{*}{ Measure } & \multicolumn{6}{|c|}{ Head-Outline Context } \\
\hline & \multicolumn{2}{|c|}{ Absent } & \multicolumn{2}{|c|}{ Congruent } & \multicolumn{2}{|c|}{ Incongruent } \\
\hline & $M$ & $S D$ & $M$ & $S D$ & $M$ & $S D$ \\
\hline Discriminability $\left(A^{\prime}\right)$ & .86 & .01 & .98 & .01 & .50 & .20 \\
\hline Hit rate & .90 & .11 & .96 & .03 & .53 & .06 \\
\hline False alarm rate & .36 & .26 & .04 & .03 & .54 & .25 \\
\hline Response bias $\left(B^{\prime \prime}\right)$ & .41 & .44 & -.02 & .40 & .04 & .24 \\
\hline
\end{tabular}


criminate direct from averted gaze in the congruent condition, rather less so when the face was absent, and their performance was poor in incongruent conditions.

A one-way repeated measures ANOVA conducted on the $A^{\prime}$ data confirmed that head context produced a significant effect on participants' performance $[F(2,32)=$ $65.75, p<.001]$. Post hoc Newman-Keuls tests $(\alpha=.05)$ confirmed that sensitivity scores were higher in the congruent condition (mean $A^{\prime}=.98$ ) than in both the absent (mean $A^{\prime}=.86$ ) and incongruent (mean $A^{\prime}=.50$ ) conditions, and that discriminability in incongruent conditions was poorer than when the face was absent.

The mean hit rates in the congruent (.96) and absent condition (.90) were also higher than in the incongruent condition (.53), indicating that a gaze stimulus which participants judged to be looking at them when presented alone or in the context of a centrally oriented face was more likely to be judged as averted when in the context of a head angled to the left or right. A repeated measures ANOVA comparing hit rates in the three conditions confirmed that context influenced performance $[F(2,32)=183.62, p<$ $.001]$. Furthermore, a planned comparison of hit rates in congruent and incongruent conditions was also significant $[t(32)=17.62, p<.001]$, suggesting that participants were indeed experiencing an illusory shift of direct gaze in this experiment.

The context manipulation also influenced false alarm rates, as can be seen in Table 3. Specifically, participants made a higher proportion of false alarms in the absent and incongruent conditions ( $M=.36$ and .54 , respectively) than in the congruent condition $(M=.04)$. A repeated measures ANOVA confirmed that context produced a significant effect on false alarm rates $[F(2,32)=27.08, p<$ $.001]$. A planned comparison also confirmed that the false alarm rate was significantly higher in the incongruent than in the congruent condition $[t(32)=7.26, p<.001]$, suggesting that averted gazes were also subject to an illusory shift caused by an incongruently rotated head outline.

The mean bias index values were also very similar to those obtained in Experiment 1A. These indicate that participants were using a neutral criterion in congruent and incongruent conditions but operating with a liberal bias when the face context was absent. One-sample $t$ tests comparing the scores with a bias score of zero provided support for these observations. Bias scores in congruent and incongruent conditions were not significantly different from zero $(p \mathrm{~s}>.5)$, but participants were operating with a significantly negative bias when the eyes were presented with no face context $[t(16)=3.82, p<.01]$.

\section{Discussion}

The results of this experiment were very similar to those obtained with full-face images in Experiment 1A. Again, participants were less able to discriminate direct from averted gaze in incongruent than in congruent images. Moreover, this reduction in discriminability could be attributed to both an increase in the false alarm rate and a decrease in hit rate when the head outline was incongruent with the gaze direction. These findings suggest that head contour alone is sufficient to induce a Wollaston-like effect and hence exerts an effect on the perception of gaze direction.

\section{EXPERIMENT 2B}

In order to examine whether or not an image-based process is responsible for the effects obtained in Experiment $2 \mathrm{~A}$, we repeated this experiment but with the stimuli rotated $180^{\circ}$. Given that the full-face images used in Experiment 1A also produced an effect on gaze perception when inverted (Experiment 1B), we expected that the effects of head contour would also persist in inverted images in this experiment.

\section{Method}

Participants. Seventeen Open University students attending summer school at the University of Stirling participated in this experiment. All reported normal or corrected-to-normal vision.

Materials, Design, and Procedure. These were identical to those of Experiment 2A, save for one detail: The full-face, eyes-only, and masking stimuli were each rotated $180^{\circ}$.

\section{Results}

Mean discriminability and bias values, hit rates, and false alarm rates are presented in Table 4 . A comparison of the sensitivity data in this table with those from Experiment 2A (Table 3) indicates that inversion seems to have had little influence on the pattern of effects. Once again, participants' discriminability scores were high in the congruent condition (mean $A^{\prime}=.94$ ), but reduced when the head context was removed (mean $A^{\prime}=.81$ ) and reduced still further when the head was incongruent with the direction of gaze (mean $A^{\prime}=.25$ ). A repeated measures ANOVA conducted on the $A^{\prime}$ data confirmed that partici-

Table 4

Means and Standard Deviations of $\boldsymbol{A}^{\prime}$ Values, Hit Rates, False Alarm Rates, and $B^{\prime \prime}$ Values Recorded in Each Condition of Experiment 2B

\begin{tabular}{|c|c|c|c|c|c|c|}
\hline \multirow[b]{3}{*}{ Measure } & \multicolumn{6}{|c|}{ Head-Outline Context } \\
\hline & \multicolumn{2}{|c|}{ Absent } & \multicolumn{2}{|c|}{ Congruent } & \multicolumn{2}{|c|}{ Incongruent } \\
\hline & $M$ & $S D$ & $M$ & $S D$ & $M$ & $S D$ \\
\hline Discriminability $\left(A^{\prime}\right)$ & .81 & .12 & .94 & .04 & .25 & .12 \\
\hline Hit rate & .89 & .09 & .91 & .06 & .24 & .21 \\
\hline False alarm rate & .48 & .28 & .12 & .10 & .57 & .22 \\
\hline Response bias $\left(B^{\prime \prime}\right)$ & .35 & .44 & .15 & .33 & -.20 & .29 \\
\hline
\end{tabular}


pants' ability to discriminate direct from averted gaze was influenced by head context $[F(2,32)=218.81, p<.001]$. Furthermore, Newman-Keuls tests $(\alpha=.05)$ revealed that all comparisons between pairs of mean $A^{\prime}$ scores were significant.

An analysis of hit rates was also conducted to examine whether participants were likely to have experienced an illusory shift of direct gaze caused by the rotation of an inverted head-outline stimulus. Mean hit rates in both congruent (.91) and absent (.89) conditions were higher than when head and gaze were incongruent (.24). A repeated measures ANOVA confirmed that context did indeed affect hit rates $[F(2,32)=143.07, p<.001]$. A planned comparison indicated that mean hit rate in the congruent condition was significantly higher than in the incongruent condition $[t(32)=14.82, p<.001]$, suggesting that participants were once again experiencing an illusory shift of direct gaze when paired with a rotated and inverted head outline.

The false alarm rates presented in Table 4 were also influenced by context manipulation. In particular, it is clear that participants made markedly more false alarms when the head context was either incongruent $(M=.57)$ or absent $(M=.48)$ than when head and gaze were congruent $(M=.12)$. This would suggest that participants were experiencing an illusory shift of averted gaze toward themselves. A repeated measures ANOVA conducted on the false alarm data yielded a significant effect of context $[F(2,32)=27.69, p<.001]$, and a planned comparison confirmed that false alarm rates in the incongruent condition were significantly higher than in the congruent condition $[t(32)=7.17, p<.001]$.

The pattern of bias scores across the three conditions with inverted heads was rather different from that with upright faces. As before, there was a bias toward responding that gaze was direct when the face context was absent. However, inversion seems to have introduced a similar, but smaller, bias with congruent stimuli, and an opposite bias (i.e., toward responding that gaze is averted) when head and gaze were incongruent. One-sample $t$ tests largely confirmed these observations. The mean bias score in the absent condition was significantly smaller than zero $[t(16)=3.26$, $p<.01]$, but the bias in the congruent condition was only marginally positive $[t(16)=1.93, p=.072]$. Participants were significantly biased toward responding that gaze was averted when head and gaze were incongruent $[t(16)=$ $2.75, p<.05]$.

\section{Discussion}

The main finding of this experiment was that, as in all the previous experiments, the context manipulation - this time of the inverted head contour-produced a significant effect on participants' ability to distinguish direct gaze from averted gaze. In particular, as with the upright head contour stimuli, $A^{\prime}$ scores were significantly lower when head contour and gaze were incongruent than when they were congruent with one another. Again, this reduction in discriminability could be attributed to both an increase in the false alarm rate, because participants mistakenly judged an averted gaze to be directed at them when it was accompanied by a head oriented in the opposite direction, and a decrease in hit rate, because an incongruent head contour towed a direct gaze to one side or the other. Inversion therefore had no influence on the Wollaston-type effect we have observed with either the full-face contexts (Experiment 1A) or head contour alone (Experiment 2A). These findings suggest that the effect arises as a result of low-level image-based processes.

The inversion manipulation did, however, introduce some bias in participants' responses. As in previous experiments, in the absence of any face context, participants tended to make more "direct" than "averted" responses while no particular bias existed in either of the other two conditions. However, here, inversion of the head contour introduced a bias toward responding that gaze was averted in the incongruent condition. It seems that, with conflicting head and gaze information, participants bias their responses toward the more salient stimulus (the head contour).

\section{EXPERIMENT 2C}

The findings of Experiments $2 \mathrm{~A}$ and $2 \mathrm{~B}$ suggest that the shape of the head contour is sufficient to influence the perception of gaze. However, it is possible that participants in these experiments were using another cue to head orientation that was present in the images. When head and gaze are directed straight ahead, the outline shape of the head is bilaterally symmetrical, and the eyes are located in the horizontal center of this shape. Now, as the head rotates, not only does the shape of the head contour deviate from bilateral symmetry, but the eyes are displaced laterally from the center of the shape bounded by the occluding contour of the head. Since the eyes were also displaced in this way in conditions with rotated heads in Experiments $1 \mathrm{~A}$ and 2A (see Figures 2 and 3), it is possible that participants were using the horizontal displacement of the eyes within the surrounding face to compute head angle, and it was this cue, rather than the shape of the head contour, that influenced the perceived direction of gaze. Indeed, Maruyama and Endo $(1983,1984)$ showed that a Wollaston-like effect could be induced in schematic faces by simply displacing the eyes alone to the left or right within a circular head outline.

In Experiment 3, therefore, the stimuli used in Experiment $2 \mathrm{~A}$ were manipulated so that the eyes always appeared in the center of the shape bounded by the face contour. If displacement of the eye region was responsible for the effects obtained in the previous experiments, we would expect no effect of head context in this experiment. On the other hand, we would expect the effect to persist if the shape of the head outline was used as a cue to head orientation, which, in turn, would influence the perception of eye gaze.

\section{Method}

Participants. Once again, 17 Open University students acted as participants in this experiment. All had normal or corrected-tonormal vision. 


\section{Head Absent}
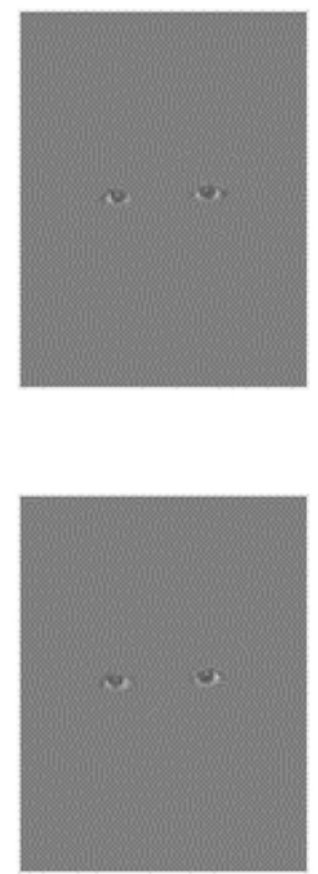

\section{Head Congruent}
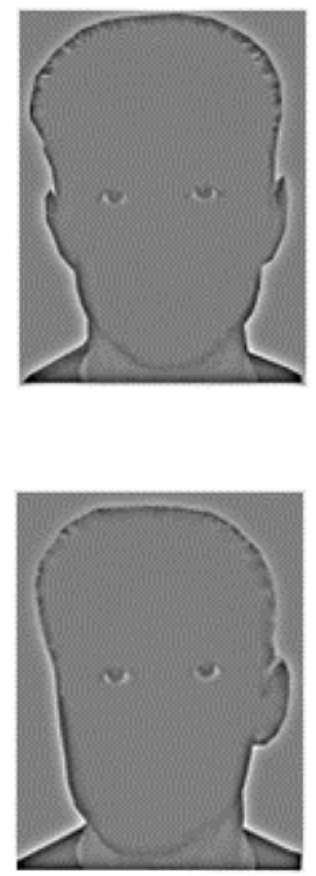

\section{Head Incongruent}
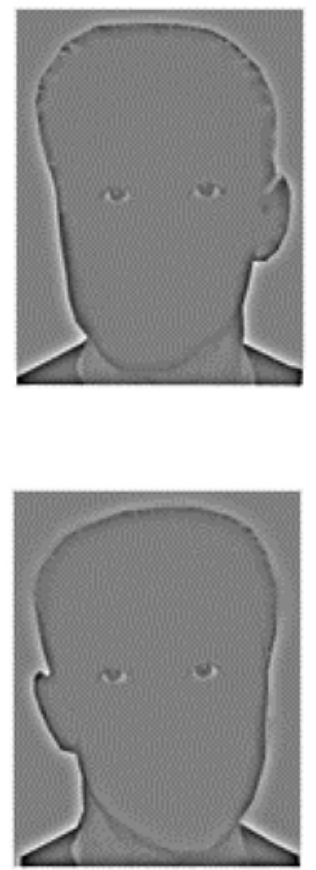

Figure 4. Reproductions of some of the stimuli used in Experiment 2C. The left column contains stimuli in the head-absent condition; the middle column, stimuli in the headcongruent condition; and the right column, stimuli in the head-incongruent condition. The upper row of stimuli have direct gazes, and those in the lower row, gazes averted to the left.

Materials, Design, and Procedure. The design and procedure remained identical to those used in the previous experiment. However, the materials used in this study differed from those used in Experiment $2 \mathrm{~A}$ in the following respect. For all the stimuli where the heads were oriented to the left or right, the eye region was shifted horizontally to offset the displacement caused by the rotation of the head. In heads rotated to the viewer's left, for example, Adobe Photoshop software was used to shift the eye region $6 \mathrm{~mm}\left(0.6^{\circ}\right)$ to the viewer's right. The eyes were shifted by the same distance to the left in heads rotated to the viewer's right. Examples of the stimuli used in this experiment are illustrated in Figure 4.

\section{Results}

Mean discriminability and bias values, hit rates, and false positive rates are presented in Table 5. The pattern of data displayed in this table is clearly very similar to that of
Experiment 2A. Participants were well able to discriminate direct from averted gaze in the congruent condition (mean $A^{\prime}=.93$ ), but their performance was slightly poorer when the head was absent (mean $A^{\prime}=.88$ ) and poorer still when head angle and gaze were incongruent (mean $A^{\prime}=$ .80). Furthermore, in the incongruent condition, participants' hit rates were lower than those in the congruent condition ( $M=.67$ and .92 , respectively) and their false alarm rates were higher ( $M=.23$ and .13 , respectively). Again, the pattern here is similar to that in the previous experiments.

A series of one-way repeated measures ANOVA's conducted on the $A^{\prime}$, hit rate, and false alarm rates confirmed these observations. First, head context produced a significant effect on participants' ability to discriminate direct gaze from averted gaze $[F(2,32)=17.31, p<.001]$.

Table 5

Means and Standard Deviations of $\boldsymbol{A}^{\prime}$ Values, Hit Rates, False Alarm Rates, and $B^{\prime \prime}$ Values Recorded in Each Condition of Experiment 2C

\begin{tabular}{|c|c|c|c|c|c|c|}
\hline \multirow[b]{3}{*}{ Measure } & \multicolumn{6}{|c|}{ Head-Outline Context } \\
\hline & \multicolumn{2}{|c|}{ Absent } & \multicolumn{2}{|c|}{ Congruent } & \multicolumn{2}{|c|}{ Incongruent } \\
\hline & $M$ & $S D$ & $M$ & $S D$ & $M$ & $S D$ \\
\hline Discriminability $\left(A^{\prime}\right)$ & .88 & .07 & .93 & .07 & .80 & .12 \\
\hline Hit rate & .87 & .14 & .92 & .06 & .67 & .20 \\
\hline False alarm rate & .31 & .15 & .13 & .16 & .23 & .16 \\
\hline Response bias $\left(B^{\prime \prime}\right)$ & .42 & .42 & -.03 & .40 & -.12 & .31 \\
\hline
\end{tabular}


Post hoc Newman-Keuls tests ( $\alpha=.05)$ confirmed that $A^{\prime}$ scores were significantly higher in the congruent condition than in both the absent and incongruent conditions and that performance was significantly poorer when head and gaze were incongruent than when the head outline was absent. Second, hit rate scores were also significantly affected by the head-context manipulation $[F(2,32)=$ $17.31, p<.001]$, and a planned comparison indicated that scores in the congruent condition were significantly higher than in the incongruent condition $[t(32)=5.47, p<.01]$. Head context also produced a significant effect on false alarm rates $[F(2,32)=9.50, p<.01]$, and a planned comparison confirmed that participants made significantly more false alarms in the incongruent condition than in the congruent condition $[t(32)=2.46, p<.05]$.

Finally, an inspection of Table 5 reveals that the pattern of bias index scores was also very similar to that obtained in Experiment 2A. Once again, participants seemed to operate with neutral criteria in congruent and incongruent conditions $(M=-.03$ and -.12 , respectively) but adopted a more liberal criterion $(M=.42)$ when the gaze stimuli were presented in the absence of the head context. A series of one-sample $t$ tests comparing the mean $B^{\prime \prime}$ scores with a neutral criterion of zero confirmed these observations. There were no significant biases in congruent or incongruent conditions ( $p s>.1)$, but there was a significant positive bias when the head context was absent $[t(16)=4.09, p<.01]$.

\section{Discussion}

The results of this experiment confirm that head outline is sufficient to induce a Wollaston-like effect on the perception of gaze direction. Even when the horizontal displacement of the eyes in rotated heads was controlled, $A^{\prime}$ scores were significantly lower when head contour and gaze direction were incongruent than when they were oriented in the same direction. As in Experiments 1 and 2, this reduction in participants' ability to discriminate direct gaze from averted gaze could be attributed to both a higher false alarm rate and a lower hit rate in incongruent than in congruent conditions.

Although the effect most certainly persisted in the absence of any displacement of the eyes, its magnitude was reduced, compared with that obtained in Experiments $1 \mathrm{~A}$ and 2A. Thus, it may well be that the horizontal displacement of the eyes within the overall face frame is used as another cue to head orientation and does indeed contribute to the perception of gaze direction as shown in the work of Maruyama and Endo $(1983,1984)$ with their schematic faces. However, the results of Experiment $2 \mathrm{C}$ confirm that the shape of the head profile is sufficient to influence the perception of gaze direction when eye displacement is controlled.

So far, we have established that the context provided by the angle of rotation of both a full face and the head contour isolated from the internal features exerts an influence on the perception of gaze. Furthermore, this influence seems to occur by virtue of some low-level image-based processes.
What of nose angle, the other major cue that Wilson et al. (2000) argue is important in head perception? Is a deviation in nose angle from vertical sufficient to influence the perception of gaze? This question was addressed in the final pair of experiments.

\section{EXPERIMENT 3A}

In this experiment, the shape of the head contour in congruent and incongruent conditions remained symmetrical (i.e., directed straight at the viewer), but the relationship between the nose angle and gaze direction was manipulated. We therefore investigated whether participants' ability to distinguish direct gaze from averted gaze was influenced by the context provided by the angle of the nose. Wilson et al. (2000) maintain that head contour and nose angle provide cues of equivalent strength for discriminating head angle. If, in order to compute gaze angle, the visual system integrates information from the same cues with information extracted from the eyes, we would also expect nose angle to influence gaze perception. Two lines of evidence hint that this might actually be the case. First, some more of Wollaston's (1824) original drawings seem to indicate that a change in the angle of the nose is sufficient to induce a change in the apparent direction of a person's gaze. Second, Maruyama and Endo (1984, Experiment 2) showed that a line denoting the profile shape of the nose, mouth, and chin could indeed influence judgments of gaze direction in schematic faces. Thus, although cues other than nose angle were available in these stimuli, participants may well have been using the deviation of nose angle from vertical as a cue to head orientation, and this cue may, in turn, have influenced the perception of gaze direction. In view of these studies, we predicted that nose angle would indeed produce effects on gaze perception similar to those observed in Experiments 1 and 2.

\section{Method}

Participants. Seventeen Open University students acted as participants in this experiment. Again, all had normal or corrected-tonormal vision.

Materials, Design, and Procedure. In this experiment, the headoutline context was held constant, directed toward the observer in all conditions. In order to achieve this, the eyes-only stimuli used in Experiment $1 \mathrm{~A}$ were pasted onto copies of the original grayscale image of the head directed toward the observer. In this way, eyes-only stimuli and full-face stimuli were created with gaze directed straight ahead, to the left, and to the right. In order to vary the nose context, the nose regions were cut from the full grayscale images of the leftward- and rightward-angled heads used in Experiment 1A. These left- and right-angled noses were then pasted onto full-face stimuli to create stimuli in which nose and gaze were congruent and incongruent. The incongruent images were created by pasting copies of the left- and right-angled noses onto the full-face images with gaze directed to the right and left, respectively, as well as onto images where the gaze was directed straight ahead. Similarly, congruent stimuli were created by pasting the left- and right-angled noses onto faces with gazes oriented to the left and right, respectively. Examples of the stimuli used in this experiment are illustrated in Figure 5, and all were identical in size to those used in Experiment $1 \mathrm{~A}$.

All other aspects of the materials, design, and procedure remained identical to those in Experiment 1A. 


\section{Nose Absent}
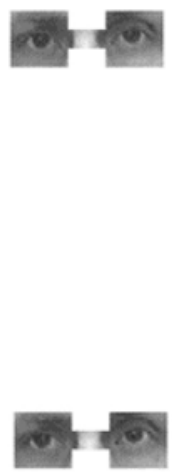
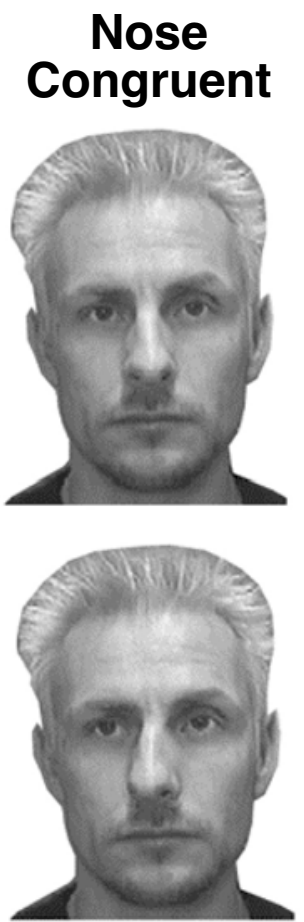

\section{Nose Incongruent}
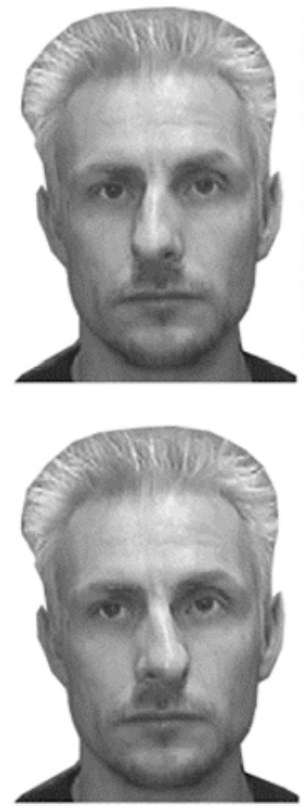

Figure 5. Reproductions of some of the stimuli used in Experiments 3A and 3B. The left column contains stimuli in the nose-absent condition; the middle column, stimuli in the nosecongruent condition; and the right column, stimuli in the nose-incongruent condition. The upper row of stimuli have direct gazes, and those in the lower row, gazes averted to the left.

\section{Results}

The means of participants' $A^{\prime}$ and $B^{\prime \prime}$ scores are summarized in Table 6 . An inspection of the discriminability data in this table reveals that the context manipulationthis time, of the nose - produced similar, but smaller, effects on participants' performance, as did the full-face and head-outline manipulations in Experiments 1A and 2A. Participants' discriminability was greatest when gaze and nose were congruent (mean $A^{\prime}=.90$ ), but fell off when the face context was removed (mean $A^{\prime}=.83$ ) and was reduced only slightly further when the nose was oriented in an incongruent direction to the eyes (mean $A^{\prime}=.80$ ). In support of these observations, a repeated measures ANOVA conducted on the $A^{\prime}$ data yielded a significant effect of nose context $[F(2,32)=5.33, p<.05]$. Post hoc NewmanKeuls tests $(\alpha=.05)$ indicated that discriminability was significantly greater in congruent than in both absent and incongruent conditions, but performance in the latter two conditions did not differ.

The hit rate scores also suggest that the nose manipulation affected participants' perception of direct gaze. Mean hit rates in absent $(M=.89)$ and congruent $(M=.85)$ conditions were higher than in the incongruent condition $(M=.74)$. A repeated measures ANOVA confirmed that these means differ $[F(2,32)=6.85, p<.01]$, and a planned comparison indicated that participants were less likely to decide that a direct gaze was looking at them when the nose was angled to one side than when it was directed straight ahead $[t(32)=2.63, p<.05]$.

Context also had a small effect on participants' false alarm rates. Of particular relevance is the observation that more false alarms were made when nose and gaze were

Table 6

Means and Standard Deviations of $\boldsymbol{A}^{\prime}$ Values, Hit Rates, False Alarm Rates, and $B^{\prime \prime}$ Values Recorded in Each Condition of Experiment 3A

\begin{tabular}{|c|c|c|c|c|c|c|}
\hline \multirow[b]{3}{*}{ Measure } & \multicolumn{6}{|c|}{ Nose Context } \\
\hline & \multicolumn{2}{|c|}{ Absent } & \multicolumn{2}{|c|}{ Congruent } & \multicolumn{2}{|c|}{ Incongruent } \\
\hline & $M$ & $S D$ & $M$ & $S D$ & $M$ & $S D$ \\
\hline Discriminability $\left(A^{\prime}\right)$ & .83 & .12 & .90 & .09 & .80 & .17 \\
\hline Hit rate & .89 & .12 & .85 & .12 & .74 & .17 \\
\hline False alarm rate & .41 & .26 & .18 & .16 & .27 & .21 \\
\hline Response bias $\left(B^{\prime \prime}\right)$ & .41 & .33 & .03 & .47 & .04 & .47 \\
\hline
\end{tabular}


incongruent $(M=.27)$ than when congruent $(M=.18)$. This suggests that nose orientation can influence the perception of an averted, as well as a direct, gaze. Indeed, a repeated measures ANOVA confirmed that nose context had a significant effect on false alarm rates $[F(2,32)=$ $17.80, p<.001]$, and a planned comparison revealed that participants were significantly more likely to misjudge that an averted gaze was actually looking directly at them when the nose was incongruent than when it was congruent with the true direction of gaze.

We will now turn to the bias data. Table 6 shows that participants set neutral criteria in the congruent and incongruent conditions, but that - as in previous experimentsthey showed a bias toward responding "direct" when the face context was removed. One-sample $t$ tests indicated that this bias was indeed significantly different from zero $[t(16)=5.23, p<.001]$ and that bias was neutral in the other two conditions $(p \mathrm{~s}>.5)$.

\section{Discussion}

The results of Experiments $1 \mathrm{~A}-2 \mathrm{C}$ suggested that the full-face and head-outline contours exert an influence on gaze perception. Similarly, the findings of Experiment 3A suggest that the deviation of nose angle alone can influence the perception of gaze direction, as suggested by Wollaston's (1824) drawings and by Maruyama and Endo's (1984) study. When the nose angle was incongruent with the true line of regard of the eyes, participants were less able to distinguish direct gaze from averted gaze than when nose and gaze were congruent. As in previous experiments, the poorer discriminability with incongruent stimuli could be attributed to both a decrease in hit rate and an increase in the rate of false alarms. Participants made fewer hits as a deviated nose towed the line of regard of a direct gaze toward the direction indicated by the nose angle. Conversely, the increased rate of false alarms could be attributed to a leftward gaze being pulled toward a nose deviated to the right - and vice versa - so participants perceived the gaze as being less averted; in other words, it was perceived as more likely to be direct.

Another point to note is that the effect of nose context is very much smaller than that of the full-face, or the headcontour manipulation in previous experiments. So, although nose deviation might, in principal, provide a cue to head direction of strength equal to the shape of the head contour (Wilson et al., 2000), the latter exerts a greater influence on gaze perception. However, we should be somewhat cautious in drawing this conclusion because - in effectnose angle was actually in competition with head orientation in this experiment (head contour remained fixed in the "direct" orientation in both congruent and incongruent trials), whereas no equivalent competition existed for the head contour in Experiment 2A. Nevertheless, regardless of the size of influence of the nose cue, the fact that nose angle has exerted an effect on gaze perception, in spite of the presence of the head-outline context, is good evidence that this cue is used in the perception of gaze direction.

Inversion of both the full-face and head-contour stimuli failed to eliminate the influence that these signals exert on gaze perception. In Experiment 3B, we asked whether the same is true of the nose angle cue.

\section{EXPERIMENT 3B}

In this experiment, the stimuli used in Experiment 3A were rotated $180^{\circ}$, and participants' ability to distinguish direct from averted gaze was again assessed. Again, if a higher level configural process is responsible for producing the effect of nose angle on gaze perception, we would expect it to be eliminated when the faces are inverted. Persistence of the effect under inverted conditions, on the other hand, would implicate a lower level image-based account. As mentioned in the discussion of Experiment 1B, Maruyama and Endo's (1984) Wollaston-like illusion was influenced by inversion of their schematic face stimuli, leading them to conclude that a configurational integration was responsible for the illusory shift in gaze. To the extent that their Wollaston effect was induced by the angle of the nose (see above), we might also expect the influence of nose angle on gaze perception to be similarly sensitive to inversion of the face stimuli.

\section{Method}

Participants. Seventeen individuals from the same population as in the previous experiments served as participants in Experiment 3B.

Materials, Design, and Procedure. The face, gaze, and masking stimuli used in Experiment $3 \mathrm{~A}$ were all rotated $180^{\circ}$; otherwise, all aspects of the design and procedure remained the same as in Experiment $3 \mathrm{~A}$.

\section{Results}

Means of participants' discriminability and bias scores in the three experimental conditions are presented in

Table 7

Means and Standard Deviations of $\boldsymbol{A}^{\prime}$ Values, Hit Rates, False Alarm Rates, and $B^{\prime \prime}$ Values Recorded in Each Condition of Experiment 3B

\begin{tabular}{|c|c|c|c|c|c|c|}
\hline \multirow[b]{3}{*}{ Measure } & \multicolumn{6}{|c|}{ Nose Context } \\
\hline & \multicolumn{2}{|c|}{ Absent } & \multicolumn{2}{|c|}{ Congruent } & \multicolumn{2}{|c|}{ Incongruent } \\
\hline & $M$ & $S D$ & $M$ & $S D$ & $M$ & $S D$ \\
\hline Discriminability $\left(A^{\prime}\right)$ & .78 & .12 & .76 & .13 & .78 & .09 \\
\hline Hit rate & .75 & .15 & .70 & .17 & .70 & .13 \\
\hline False alarm rate & .36 & .23 & .34 & .17 & .32 & .16 \\
\hline Response bias $\left(B^{\prime \prime}\right)$ & .05 & .32 & .03 & .30 & .01 & .29 \\
\hline
\end{tabular}


Table 7. From this table, it is clear that with inverted stimuli, nose context did not greatly influence participants' ability to discriminate direct gaze from averted gaze. Indeed, a repeated measures ANOVA comparing $A^{\prime}$ scores across the three conditions failed to yield an effect of context $[F(2,32)=0.43, p=.66]$.

Nose context also appears to have exerted little effect on hit rates or false alarm rates, observations confirmed by separate repeated measures ANOVAs conducted on these data, neither of which approached statistical significance $(p s>.2)$.

Although, once again, participants operated with a rather more liberal bias in the absent condition than in the congruent and incongruent conditions, the bias scores were very close to zero throughout. One-sample $t$ tests confirmed that none of the bias scores differed significantly from zero $(p s>.4)$.

In order to compare the effects of nose context on gaze discriminability with upright and inverted stimuli, an omnibus ANOVA was conducted on the $A^{\prime}$ data from this and the previous experiment. Context (absent, congruent, and incongruent) was entered as a repeated measures factor and orientation (upright and inverted) as a betweensubjects factor. This analysis yielded a marginally significant effect of orientation with better discrimination of upright as opposed to inverted gaze stimuli $[F(1,32)=3.99$, $p=.054]$ and a significant interaction between orientation and context $[F(2,64)=4.82, p<.05]$. Simple main effects analysis confirmed that context exerted an effect on discriminability scores for upright $(p<.05)$ but not for inverted stimuli $(p=.56)$.

\section{Discussion}

In Experiments 1B and 2B, the influence of head context on gaze perception was found to persist when the head/gaze stimuli were inverted. In contrast, the results of this experiment indicate that the influence of nose angle is eliminated under inverted conditions. While some kind of image-based process seems to be responsible for the effects exerted by head contour, a rather different accountperhaps based on the encoding of spatial relations between face features - is implicated for the influence that nose angle exerts on gaze perception.

\section{GENERAL DISCUSSION}

The aim of the experiments reported here was to investigate whether the cues that are thought to be used in the perception of head orientation - the deviation of the head profile from bilateral symmetry and the deviation of nose angle from vertical - are also those that influence the perception of eye gaze. In Experiment 1A, we confirmed that the orientation of the head and internal face features can indeed influence the perception of gaze. Participants' perceptions of both direct and indirect gazes were towed in the direction of an incongruently oriented head so that a direct gaze was judged to be averted, and an averted gaze more likely to be judged as direct. Moreover, the effect on gaze discriminability was found to be uninfluenced by inversion of the stimuli (Experiment 1B), suggesting that the locus of the effect was at an early stage of processing, prior to categorization of the stimuli as faces. The remaining experiments attempted to isolate the cues responsible for the effect. In Experiment 2A, stimuli consisting of only the outline-head contour gave rise to a pattern of effects on gaze perception nearly identical to that for the full-face images. This pattern was maintained when the stimuli were inverted (Experiment 2B) and, although reduced in magnitude, persisted when the eyes were always located in the center of the surrounding face pattern (Experiment 2C). As for the second cue to head orientation-the deviation of nose angle from vertical-Experiment $3 \mathrm{~A}$ showed that this cue also influenced participants' ability to distinguish between direct and averted gazes. Although the magnitude of the effect was much smaller than that exerted by the headcontour images in the previous experiments, the pattern was identical. Finally, in contrast to the findings of previous experiments, the influence of nose angle on gaze judgments was eliminated when the stimuli were inverted (Experiment 3B), implicating the operation of a higher level mechanism perhaps based on the configural/relational encoding of the face stimuli.

Our results suggest that the cues deemed important by Wilson et al. (2000) for judging another's head angle are also capable of influencing the perception of gaze, although they seem to do so in rather different ways: head contour via a low-level process and nose angle at a later stage in processing. In the remainder of this section, we discuss each of these mechanisms before turning to more general issues concerning the role of head orientation in social interactions.

\section{Image-Based Processing of Head Orientation and Gaze Direction}

Wilson et al.'s (2000) work, together with findings of Watt (1999), Ricciardelli et al. (2000), and Sinha (2000), suggests that both head orientation and gaze direction can be coded very early in processing by mechanisms that are insensitive to inversion. For example, Wilson et al. show how head shape can be coded from the visual image by V4 units that are sensitive to concentric and radial structures. When the head is oriented at $0^{\circ}$ (i.e., looking directly toward an observer), the outputs from each of a number of these units arranged in a hexagonal array encode the overall head shape and the vertical axis of face elongation. Moreover, Wilson et al. showed how the responses of these units are bilaterally symmetric about this axis. As the face turns, the relative pooled responses of the units to the right of the axis of elongation will differ from those to the left so that a ratio describing the degree of asymmetry can be computed. Wilson et al. showed that such a ratio has a linear relationship with the angle of deviation of the head from $0^{\circ}$ to $23^{\circ}$. Thus, the symmetry axis and angles of deviations of the head outline can be extracted early in processing from the image of the face, ${ }^{1}$ a procedure that does not require the categorization of the face as such, nor the 
localization or explicit categorization of any face features. Since these V4 units essentially operate as asymmetry detectors, inversion would not be expected to affect judgments of head orientation based on this cue; the symmetry, or the deviation from symmetry, is maintained in inverted images. Indeed, Wilson et al.'s data indicate that perception of head orientation, as signaled by a combination of head contour and internal face features, was unaffected by the inversion manipulation. Our data go one step further in indicating that the influence that head contour exerts on gaze perception is unaffected by inversion.

There is a suggestion that the cues to eye-gaze direction can also be extracted very early in processing. The fact that contrast negation has an effect on judgments of gaze direction (Ricciardelli et al., 2000; Sinah, 2000) points toward an image-based, rather than a purely spatially based, representation of eye gaze. As described earlier, Watt (1999; see Langton, Watt, \& Bruce, 2000) also favors an imagebased account. He has argued that the contrast in luminance between the areas of sclera on either side of the iris provides a reliable cue that the visual system might use to determine gaze direction. Furthermore, he showed how this information could be extracted from the image of the eye by vertically oriented simple cells in striate cortex. As with the computation of head angle, this method of determining gaze direction would proceed equally well with inverted as with upright stimuli.

Thus, both head and gaze direction can, in principle, be computed early in processing by mechanisms that would be insensitive to inversion of the stimuli. Given our extraction of the relevant information concerning gaze direction and head angle, presumably some kind of additive (e.g., Cutting, Bruno, Brady, \& Moore, 1992) or multiplicative (e.g., Massaro \& Friedman, 1990) interaction takes place combining information from the two cues. An important point to note is that with these kinds of integrative interactions, the integrity of the component signals is lost; that is, a new representation of gaze direction is created from the combination of eye and head angle. This seems appropriate in the case of head contour and eye-gaze direction since the same eye stimuli can give rise to two different percepts of gaze direction, depending on the congruity or incongruity of the head contour. Whatever the precise nature of this interaction, as with the extraction of information from the component cues, it is also presumably insensitive to face inversion.

\section{Configural Processing and Nose Angle}

What of the influence of nose angle on the perception of gaze? The effect we noted in Experiment 3A was very much smaller than that exerted by the head shape in Experiment $2 \mathrm{~A}$ and was actually eliminated when the stimuli were inverted. As suggested earlier, the reduced magnitude of the effect could have been caused by the fact that head orientation and nose angle were effectively in competition in this experiment. It is therefore difficult for us to draw any firm conclusions about the relative ability of nose angle and head-shape cues to influence the percep- tion of gaze, and it also makes our interpretation of the inversion effect rather more circumspect. However, in view of the fact that Maruyama and Endo's (1984) illusionalso probably triggered by a nose-angle cue-was similarly sensitive to inversion, we suggest that the influence of nose angle on gaze judgments is unlikely to be the result of some early integration of information extracted from the image of the face, simply because the relevant image features will still be present in the inverted stimuli. The implication is that the effect arises at a later stage in processing. Another possibility, consistent with this suggestion, is that the sensitivity of the nose effect to inversion could be caused by a difficulty in actually encoding the relevant face feature - in this case, the nose - because of our unfamiliarity with upside-down faces. However, as discussed earlier, the evidence suggests that face features (nose, eyes, mouth, etc.) are, in fact, encoded in inverted faces, whereas the relationship between these features is not (e.g., Leder \& Bruce, 1998, 2000; Searcy \& Bartlett, 1996). In view of this, a more likely explanation for the inversion effect is that the nose contributes to the perception of gaze direction as part of a configuration of face features.

The term configuration is somewhat vague and has been used in rather different ways by different researchers. Holistic processing of gaze direction - an extreme version of the configural processing view (e.g., Tanaka \& Farah, 1993) — would imply that neither the nose angle nor eye direction is represented separately, but that some kind of gestalt involving the internal face features signals the direction of attention. Alternatively, a somewhat less extreme "relational" processing view (e.g., Diamond \& Carey, 1986) would imply that nose, eyes, and so forth are represented but that the processing of, say, the nose provides some kind of contextual modulation of the processing of gaze direction (e.g., Phillips \& Singer, 1997). This form of interaction can be contrasted with the additive or multiplicative interactions suggested as operating to combine head outline and gaze direction. In the latter types of interaction, information from the two sources is actually integrated to create a new representation. Contextual modulation, on the other hand, does not involve an actual integration of signals but rather a facilitation of the processing of one variable (e.g., eye direction) by information in another processing channel (e.g., nose angle). Thus, it is possible that early in processing, some kind of integrative interaction operates to combine head outline and eyegaze direction to yield a new representation specifying gaze direction. The processing of this information might then be modulated at a later stage by the context provided by the orientation of the nose.

However, it may also be the case that the computation of nose angle itself involves a kind of configural/relational processing, so that face inversion may have disrupted this process as well as - or instead of - the contextual interaction between nose angle and eye direction. Configural or relational processing may be involved in the extraction of nose angle because, in order to give a reliable indication of head 
rotation, the deviation of angle of the nose must be computed in relation to the vertical axis of elongation of the face, and not simply as the deviation of the nose angle from vertical in space (Wilson et al., 2000). In order to see that this must be so, consider a deviation in the nose angle to the viewer's left. This could signal that the head is turned to the left (rotation in the horizontal plane), or that the head is tilted to the viewer's right (rotation in the coronal plane). The estimation of head angle using the nose as a cue thus involves location of the nose region, ${ }^{2}$ a computation of the vertical axis of elongation of the face, and, of course, a computation of the nose angle itself. It seems that this process is not as simple as the coding of head angle and involves a good deal of relational processing, the kind of activity thought to be disrupted by face inversion.

To summarize, we speculate that head outline operates to influence eye direction at a very early stage in processing, possibly through some kind of integrative combination of information extracted from the visual image concerning head-outline asymmetry and scleral contrast. Nose angle, on the other hand, seems to influence the processing of gaze direction through a configural interaction at a later stage in processing after the integration of head and gaze information. However, the precise nature of these interactions awaits further research.

We have shown that head angle, as signaled by whole face, head outline, and nose angle, can influence the perception of eye-gaze direction. The choice of head and gaze angles in the present experiments was deliberately made to produce the Wollaston illusion best with the full-face images; however, further work should explore whether an interaction exists over a range of head and gaze angles. Wilson et al. speculate that as head angles approach $30^{\circ}$, deviations of the head profile from bilateral symmetry might be ineffective in coding head angle and that nose angle might be the principal cue under these conditions. Thus, it may be that with greater incongruities between head and eye-gaze angle, the nose angle will exert a larger effect on gaze perception.

Although this article has focused on the relationship between the perception of head orientation and that of eye gaze, we should be mindful of the importance of the former as an independent social signal in its own right, and not simply as a vehicle for the eyes. We have already mentioned how the head acts as the primary cue to attention direction in infants and many nonhuman primates. However, perhaps it also serves as a "special" cue in adults. Research has shown that a speaker engaged in conversation will often avert his or her gaze from a partner, only to return, for example, at the end of that speaker's conversational turn (Kendon, 1967). However, during this aversion of gaze, it may be critical for the listener to maintain attention on the speaker's face in order to process more efficiently the facial expressions and gestures or changes in face and mouth shape that can help disambiguate speech sounds (e.g., McGurk \& MacDonald, 1976). Perhaps a speaker holds a listener's attention by ensuring that the ori- entation of his or her head does not stray too far from the line of regard of the listener, even though the listener's actual eye gaze might. If this is true, the implication is that the angle of the head might actually be the more powerful cue to the direction of another's "social," as opposed to "visual," attention direction.

\section{REFERENCES}

Ando, S. (2002). Luminance-induced shift in the apparent direction of gaze. Perception, 31, 657-674.

Anstis, S. M., May HeW, J. W., \& Morley, T. (1969). The perception of where a face or television "portrait" is looking. American Journal of Psychology, 82, 474-489.

BARON-COHEN, S. (1995). The Eye Direction Detector (EDD) and the Shared Attention Mechanism (SAM): Two cases for evolutionary psychology. In C. Moore \& P. Dunham (Eds.), Joint attention: Its origins and role in development (pp. 41-59). Hillsdale, NJ: Erlbaum.

Barton, J. J. S., KeEnan, J. P., \& Bass, T. (2001). Discrimination of spatial relations and features in faces: Effects of inversion and viewing duration. British Journal of Psychology, 92, 527-549.

Bentin, S., Allison, T., Puce, A., Perez, E., \& McCarthy, G. (1996). Electrophysiological studies of face perception in humans. Journal of Cognitive Neuroscience, 8, 551-565.

BRUCE, V., \& LANGTON, S. (1994). The use of pigmentation and shading information in recognising the sex and identities of faces. Perception, 23, 803-822.

BRUCE, $\overline{\mathrm{V}}$, \& Y YOUNG, A. (1998). In the eye of the beholder: The science of face perception. Oxford: Oxford University Press.

BUTTERWORTH, G., \& JARRETT, N. (1991). What minds have in common is space: Spatial mechanisms serving joint visual attention in infancy. British Journal of Developmental Psychology, 9, 55-72.

BYRNE, R. W., \& Whiten, A. (1991). Computation and mindreading in primate tactical deception. In A. White (Ed.), Natural theories of mind: Evolution, development and simulation of everyday mindreading (pp. 127-141). Oxford: Blackwell.

Campbell, R., Heywood, C. A., Cowey, A., Regard, M., \& Landis, T. (1990). Sensitivity to eye gaze in prosopagnosic patients and monkeys with superior temporal sulcus ablation. Neuropsychologia, 28, 1123 1142.

Carey, S., \& Diamond, R. (1977). From piece-meal to configural representation of faces, Science, $195,312-314$.

Cline, M. G. (1967). The perception of where a person is looking. American Journal of Psychology, 80, 41-50.

CORKUM, V., \& MOORE, C. (1995). Development of joint visual attention in infants. In C. Moore \& P. J. Dunham (Eds.), Joint attention: Its origins and role in development (pp. 61-83). Hillsdale, NJ: Erlbaum.

Cutting, J. E., Bruno, N., Brady, N. P., \& Moore, C. (1992). Selectivity, scope, and simplicity of models: A lesson from fitting judgments of perceived depth. Journal of Experimental Psychology: General, 121, 364-381.

DIAMOND, R., \& CAREY, S. (1986). Why faces are and are not special: An effect of expertise. Journal of Experimental Psychology: General, 115, 107-117.

EIMER, M. (2000). Effects of face inversion on the structural encoding and recognition of faces: Evidence from event-related brain potentials. Cognitive Brain Research, 10, 145-158.

Emery, N. J., Lorincz, E. N., Perrett, D. I., Oram, M. W., \& BaKer, C. I. (1997). Gaze following and joint attention in rhesus monkeys (Macaca mulatta). Journal of Comparative Psychology, 111, 286-293.

GiBSON, J. J., \& Pick, A. (1963). Perception of another person's looking. American Journal of Psychology, 76, 86-94.

Goldstone, R., Medin, D., \& Gentner, D. (1991). Relational similarity and the non-independence of features in similarity judgements. Cognitive Psychology, 23, 222-262.

HANCOCK, P. J. B., BRUCE, V., \& BURTON, A. M. (1998). A comparison of two computer-based face identification systems with human perception of faces. Vision Research, 38, 2277-2288. 
Hancock, P. J. B., Burton, A. M., \& Bruce, V. (1996). Face processing: Human perception and principle components analysis. Memory \& Cognition, 24, 26-40.

HeYwood, C. A., \& Cowey, A. (1992). The role of the "face cell" area in the discrimination and recognition of faces by monkeys. Philosophical Transactions of the Royal Society of London: Series $\overline{B, \mathbf{3 3 5}_{2}}$ 31-38.

HofFMAN, E. A., \& Haxby, J. V. (2000). Distinct representations of eye gaze and identity in the distributed neural system for face perception. Nature Neuroscience, 3, 80-84.

ItAKURA, S., \& ANDERSON, J. R. (1996). Learning to use experimentergiven cues during an object-choice task by a capuchin monkey. $\mathrm{Cur}$ rent Psychology of Cognition, 15, 103-112.

Jenkins, J., \& Langton, S. R. H. (2003). Configural processing in the perception of eye-gaze direction. Perception, 32, 1181-1188.

KENDON, A. (1967). Some functions of gaze direction in social interaction. Acta Psychologica, 26, 22-63.

KLeinke, C. L. (1986). Gaze and eye contact: A research review. Psychological Bulletin, 100, 78-100.

LANGTON, S. R. H. (2000). The mutual influence of gaze and head orientation in the analysis of social attention direction. Quarterly Journal of Experimental Psychology, 53A, 825-845.

LANGTON, S. R. H., \& BRUCE, V. (1999). Reflexive visual orienting in response to the social attention of others. Visual Cognition, 6, 541-567.

Langton, S. R. H., \& Bruce, V. (2000). You must see the point: Automatic processing of cues to the direction of social attention. Journal of Experimental Psychology: Human Perception \& Performance, 26, 747-757.

LANGTON, S. R. H., Watt, R. J., \& Bruce, V. (2000). Do the eyes have it? Cues to the direction of social attention. Trends in Cognitive Sciences, 4, 50-59.

LEDER, H., \& BRUCE, V. (1998). Local and relational aspects of face distinctiveness. Quarterly Journal of Experimental Psychology, $\mathbf{5 1 A}_{2}$ 449-473.

LEDER, H., \& BRUCE, V. (2000). When inverted faces are recognised: The role of configural information in face recognition. Quarterly Journal of Experimental Psychology, 53A, 513-536.

LORD, C., \& HAITH, M. M. (1974). The perception of eye contact. Perception \& Psychophysics, 16, 413-416.

Maruyama, K., \& Endo, M. (1983). The effect of face orientation upon apparent direction of gaze. Tohoku Psychologica Folia, 42, 126-138.

MaRUYAMA, K., \& Endo, M. (1984). Illusory face dislocation effect and configurational integration in the inverted face. Tohoku Psychologica Folia, 43, 150-160.

Massaro, D. W., \& Friedman, D. (1990). Models of integration given multiple sources of information. Psychological Review, 97, 225-252.

Maurer, D., Le Grand, R., \& Mondloch, C. J. (2002). The many faces of configural processing. Trends in Cognitive Sciences, 6, 255-260.

MCGurK, H., \& MACDonalD, J. (1976). Hearing lips and seeing voices. Nature, 264, 746-748.

MoOre, C., \& CORKum, V. (1998). Infant gaze following based on eye direction. British Journal of Psychology, 16, 495-503.

O'Toole, A. J., Deffenbacher, K. A., Valentin, D., \& Abdi, H. (1994). Structural aspects of face recognition and the other-race effect. Memory \& Cognition, 22, 208-224.

Perrett, D. I., Smith, P. A. J., Potter, D. D., Mistlin, A. J., Head, A. S., Milner, A. D., \& JeEves, M. A. (1985). Visual cells in the temporal cortex sensitive to face view and gaze direction. Proceedings of the Royal Society of London: Series B, 223, 293-317.
Phillips, W. A., \& Singer, W. (1997). In search of common foundations for cortical computation. Behavioral and Brain Sciences, 20, 657-722. Ricciardelli, P., Baylis, G., \& Driver, J. (2000). The positive and negative of human expertise in gaze perception. Cognition, 77, B1-B14.

Rossion, B., Delvenne, J.-F., Debatisse, D., Goffaux, V., Bruyer, R., Crommelinck, M., \& Guérit, J.-M. (1999). Spatio-temporal localisation of the face inversion effect: An event related potentials study. Biological Psychology, 50, 173-189.

SCAIFE, M., \& BRUNER, J. S. (1975). The capacity for joint visual attention in the infant. Nature, 253, 265-266.

SeARCY, J. H., \& BARTLETT, J. C. (1996). Inversion and processing of component and spatial-relational information in faces. Journal of Experimental Psychology: Human Perception \& Performance, 22, 904915.

Sergent, J. (1984). An investigation into component and configural processes underlying face perception. British Journal of Psychology, 75, 221-242.

SINHA, P. (2000). Here's looking at you, kid. Perception, 29, 1005-1008.

SNODGRASs, J. G., \& CoRwIn, J. (1988). Pragmatics of measuring recognition memory: Applications to dementia and amnesia. Journal of Experimental Psychology: General, 117, 34-50.

TANAKA, J. W., \& FARAH, M. J. (1993). Parts and wholes in face recognition. Quarterly Journal of Experimental Psychology, 46A, 225-245.

Turk, M., \& Pentland, A. (1991). Eigenfaces for recognition. Journal of Cognitive Neuroscience, 3, 71-86.

VALENTINE, T., \& BRUCE, V. (1986). The effect of race, inversion and encoding activity upon face recognition. Acta Psychologica, 61, 259-273.

VeCERA, S. P., \& Johnson, M. H. (1995). Gaze detection and the cortical processing of faces: Evidence from infants and adults. Visual Cognition, 2, 59-87.

voN GRÜNAU, M., \& Anston, C. (1995). The detection of gaze: A stare in the crowd effect. Perception, 24, 1297-1313.

WATT, R. J. (1999, August). What your eyes tell my eyes, and how your eyebrows try to stop them. Paper presented at the Tenth International Conference on Perception and Action, University of Edinburgh.

Wilson, H. R., Wilkinson, F., Lin, L.-M., \& Castillo, M. (2000). Perception of head orientation. Vision Research, 40, 459-472.

YIN, R. K. (1969). Looking at upside down faces. Journal of Experi$\underline{\text { mental Psychology, 81, } 141 .}$

\section{NOTES}

1. Hancock, Bruce, and Burton (1998) have serendipitously shown that principle components analysis (PCA) of image pixel values can encode the angle of the head. PCA is a technique that extracts statistical regularities in a set of images and can encode various facial dimensions such as identity, expression, and gender with, it is claimed, some psychological plausibility (e.g., Hancock, Burton, \& Bruce, 1996; O’Toole, Deffenbacher, Valentin, \& Abdi, 1994; Turk \& Pentland, 1991).

2. The location of face features is, itself, a far from trivial problem. However, Wilson et al. (2000) suggest that location of the bridge of the nose region could, in principle, be achieved by V4 units before the sampling of orientation-specific cells below this point could code its angle of deviation from vertical.

(Manuscript received June 18, 2002; revision accepted for publication October 5, 2003.) 